\title{
La sostenibilidad fiscal y la política de balance cíclicamente ajustado: metodología y análisis para Chile
}

\author{
Mauricio G. Villena, Cristóbal Gamboni y Andrés Tomaselli
}

\section{Resumen}

Este trabajo presenta un marco de análisis de la sostenibilidad fiscal para la economía chilena. Primero, se aborda el cálculo ex post de indicadores de vulnerabilidad y sostenibilidad fiscal, sobre la base de la estimación de un nivel de deuda sostenible, considerando un estado estacionario de las finanzas públicas. Segundo, se desarrolla un modelo dinámico de sostenibilidad fiscal. En este estudio se presenta un modelo ad hoc para las finanzas públicas chilenas, siendo el primero en incorporar la dinámica del Fondo de Reserva de Pensiones (FRP), con sus reglas de acumulación y desembolso, y la política de balance cíclicamente ajustado. Finalmente, se simula la senda de deuda neta del gobierno central presupuestario de Chile hacia 2025, utilizando las proyecciones realizadas en el Informe de Finanzas Públicas 2018, evaluando un escenario macroeconómico de tendencia y otro adverso, todo esto en el contexto de la regla de balance cíclicamente ajustado.

\section{Palabras clave}

Administración fiscal, política fiscal, hacienda pública, macroeconomía, deuda pública, indicadores económicos, tendencias económicas, Chile

\section{Clasificación JEL}

E62, H6, O23, O11

\section{Autores}

Mauricio G. Villena es Profesor Titular en la Escuela de Negocios de la Universidad Adolfo Ibáñez, Santiago. Correo electrónico: mauricio.villena@uai.cl.

Cristóbal Gamboni es Economista Senior en el Servicio de Estudios del Banco BBVA, Chile. Correo electrónico: cristobal.gamboni@bbva.com.

Andrés Tomaselli es Magíster en Políticas Públicas de la Universidad de Chile. Correo electrónico: aptomaselli@gmail.com. 


\section{Introducción}

Los elevados y crecientes niveles de endeudamiento generan un deterioro de la calificación crediticia, con el consiguiente aumento de la incertidumbre en los mercados financieros y el incremento de la prima de riesgo, situación que implica que un país deba destinar mayores recursos para el pago del servicio de su deuda, presionando aún más al gasto. Por lo tanto, en muchos casos, la única vía para recortar el déficit en el corto plazo es mediante ajustes del gasto público, con negativas consecuencias sociales.

Efectivamente, la dinámica y la sostenibilidad de la deuda pública dependen del comportamiento del déficit público y su interrelación con las diferentes fases del ciclo económico, su vulnerabilidad o volatilidad frente a eventos exógenos, los mercados de capitales, las tasas de interés tanto internas como externas y el tipo de cambio real. Así, la deuda pública neta representa la acumulación de resultados fiscales pasados, mientras que los balances futuros incorporan los ingresos y gastos por intereses resultantes. De esta manera, los desequilibrios fiscales sistemáticos se traducirán en presiones futuras de gasto en intereses, los que contribuirán, a su vez, a la acumulación de nueva deuda.

Durante cerca de dos décadas, la política fiscal chilena se ha basado en el concepto de balance cíclicamente ajustado del gobierno central total, que es consistente con los equilibrios macroeconómicos de mediano plazo, es decir, con un nivel de deuda pública sostenible en el tiempo ${ }^{1}$. En la práctica, hasta 2010, esto implicó una tendencia decreciente en la acumulación de pasivos brutos por parte del gobierno central presupuestario², lo cual tiende a revertirse en los últimos años, pero sin recuperar los niveles de los años anteriores a la regla. Como se puede inferir del gráfico 1.A, en promedio la razón deuda bruta/PIB pasa de un 22,6\% en el período 1990-2000 a un 11,1\% en el período 2001-2016.

\section{Gráfico 1}

Deuda del gobierno central, 1990-2016

\section{A. Activo financiero y pasivo financiero bruto total} del gobierno central, 1990-2016

(en porcentajes del PIB)

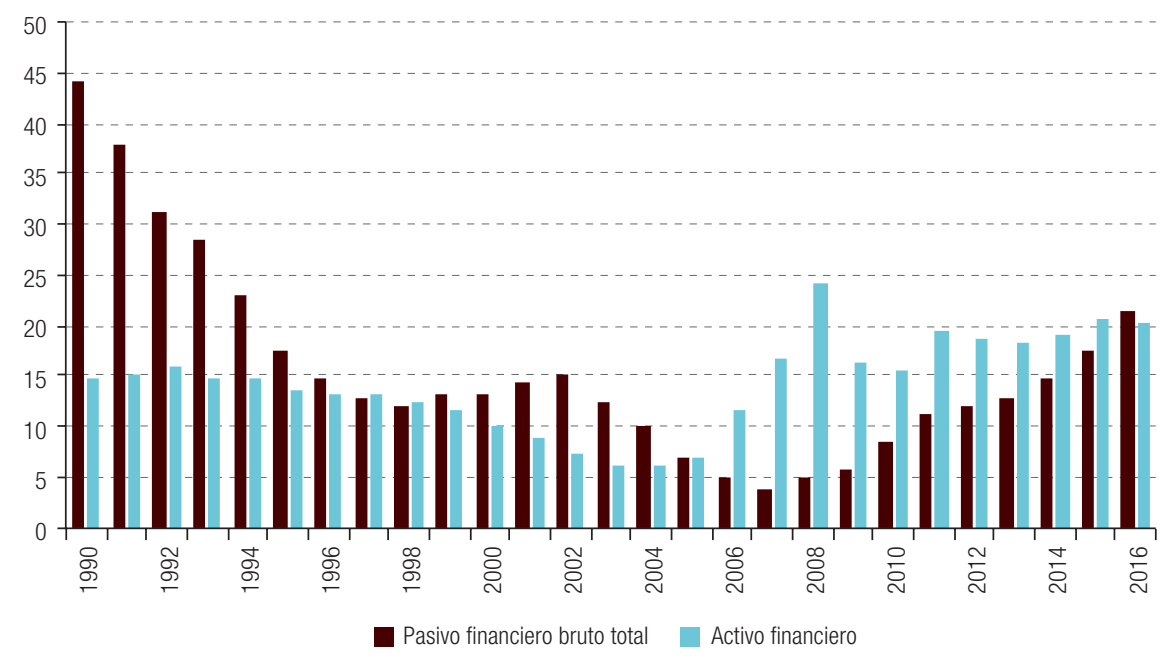

\footnotetext{
1 El gobierno central total abarca al conjunto de instituciones sometidas a las mismas normas de planificación y ejecución presupuestaria, que guardan estrecha relación de dependencia con el Poder Ejecutivo, más las operaciones extrapresupuestarias ligadas a este nivel de gobierno.

2 En este estudio, al tratar los flujos de ingresos y gastos se considera la cobertura del gobierno central total, mientras que al analizar los acervos tanto de deuda bruta como de deuda neta se abarca la cobertura del gobierno central presupuestario. Este último omite los bonos de reconocimiento no liquidados por razones que se explican más adelante.
} 
B. Pasivo financiero neto del gobierno central, 1990-2016

(en porcentajes del PIB)

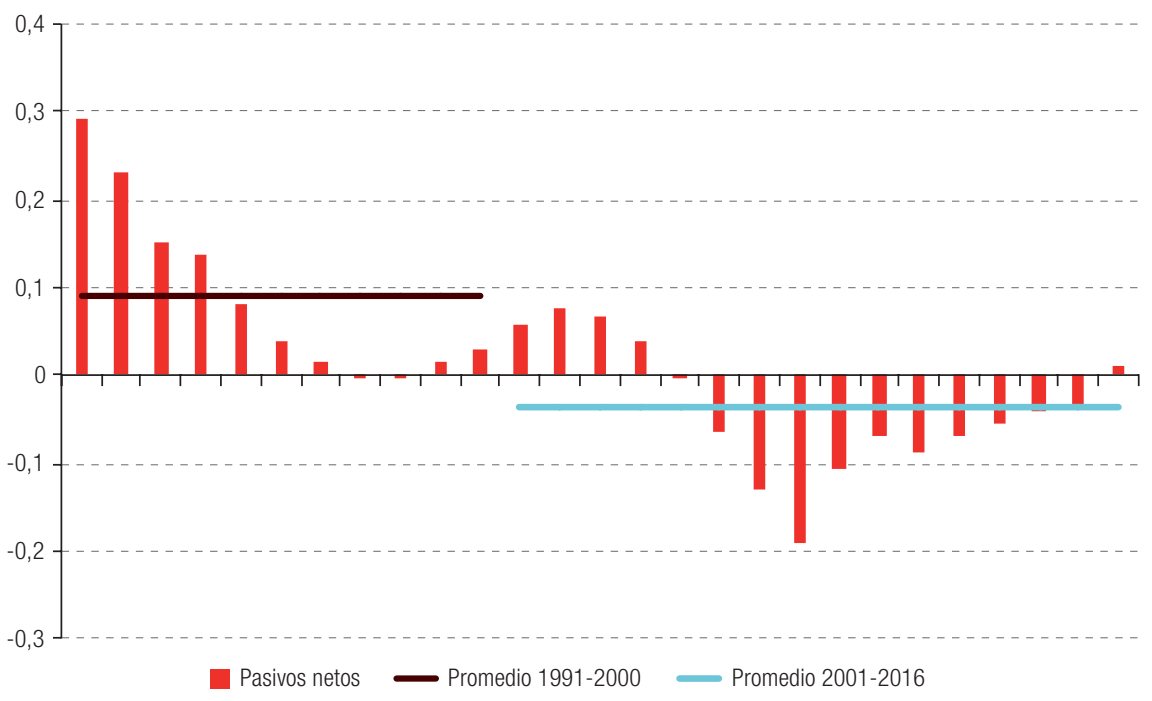

Fuente: Elaboración propia, sobre la base de información del Ministerio de Hacienda y el Banco Central de Chile.

Así, durante la operación de la regla fiscal de balance cíclicamente ajustado (BCA), el gobierno central presupuestario pasó de una posición deudora neta en 2000 a una posición acreedora neta desde 2005, situación que se mantiene hasta 2015 (véase el gráfico 1.B). Esta evolución del endeudamiento neto ha permitido, entre otras cosas, mejorar su credibilidad como emisor de deuda, tanto a nivel local como internacional.

A pesar de los logros de la política fiscal, Chile ha enfrentado una importante presión sobre las cuentas fiscales en la última década. En primera instancia, la crisis financiera internacional de 2008-2009 y el terremoto y tsunami de febrero de 2010. Adicionalmente, en años recientes la política fiscal ha estado expuesta a una serie de desequilibrios que han llevado al país a completar cinco años con déficits de presupuesto, con un consecuente aumento de la deuda. De hecho, debido al sostenido deterioro de la situación fiscal, después de 12 años Chile ha pasado en 2016 de acreedor neto a deudor neto, con una deuda neta del 1\% del PIB. Dicha posición fiscal nos retrotrae al año 2004, cuando la deuda neta representaba un 4\% del PIB. Esto es consistente con el aumento que ha mostrado la deuda bruta, que en 2016 alcanzó al 21,3\% del PIB, su mayor nivel desde 1994, cuando este indicador ascendió al 22,9\% del PIB (véase el cuadro 1). Como consecuencia del aumento de la deuda neta, el gasto en intereses ya ha aumentado más de 1.000 millones de dólares comparado, por ejemplo, con el gasto de 2009 (DIPRES, 2017d) ${ }^{3}$. Este deterioro de las cuentas fiscales ya ha sido internalizado por las clasificadoras de riesgo, que han bajado la calificación del país: la agencia Standard \& Poor's bajó la clasificación de riesgo en moneda extranjera a largo plazo de Chile de AA-, obtenida en 2012, a A+, en lo que constituye la primera rebaja de la nota del país en 25 años (véase Arellano, 2017, pág. 14).

Aunque los actuales niveles de deuda neta del gobierno central de Chile no parecen demasiado altos al compararlos con los países de la región o de la OCDE, si se consideran las presiones de gasto que se proyectan para los próximos años la posición fiscal podría quedar en una situación cada vez más vulnerable. En este contexto, una pregunta que surge es, dado este escenario, en qué medida está comprometida la sostenibilidad fiscal de largo plazo de Chile, ante potenciales turbulencias de la economía.

3 Véase DIPRES (2017d). 
Este trabajo busca contribuir a la discusión en esta línea, presentando un marco de análisis de la sostenibilidad fiscal para la economía chilena. Para ello, en primer término, se aborda el cálculo ex post de indicadores de vulnerabilidad y sostenibilidad fiscal adaptados a la realidad fiscal chilena, sobre la base de la estimación de un nivel de deuda sostenible, considerando un estado estacionario de las finanzas públicas. De esta forma, es posible evaluar de manera cuantitativa la evolución de la sostenibilidad fiscal chilena y, también, entregar una perspectiva del impacto de las crisis recientes.

En segundo término, dado que el análisis de indicadores es estático y no permite simular los potenciales efectos de una crisis económica sobre las finanzas públicas chilenas, en este trabajo se desarrolla un modelo dinámico de sostenibilidad fiscal. Particularmente, este estudio contribuye a la literatura desarrollando un modelo ad hoc para las finanzas públicas chilenas, siendo el primero en incorporar la dinámica del Fondo de Reserva de Pensiones (FRP) constituido en 2006, con sus reglas de acumulación y desembolso, y la política de balance estructural actualmente vigente para Chile (Larraín y otros, 2011; DIPRES, 2017a).

Por último, a fin de ejemplificar el funcionamiento del modelo dinámico propuesto, se simula la senda de deuda neta del gobierno central presupuestario de Chile hacia 2025, evaluando un escenario macroeconómico de tendencia y otro adverso, todo esto en el contexto de la regla de balance cíclicamente ajustado. El escenario de tendencia considera como posición inicial el presupuesto 2018 y la proyección financiera del sector público para el período 2019-2021 (véase DIPRES (2017b)) y una convergencia a sus valores de tendencia. En el escenario adverso también se considera como base la proyección financiera del sector público, pero a partir de 2018 se simula una crisis de demanda externa que repercute fuertemente en el crecimiento del producto para los siguientes años. Después de la desaceleración de la actividad, se asume un período de recuperación progresiva hasta alcanzar la convergencia a los valores de tendencia hacia finales del período proyectado.

El documento se estructura como sigue. En la sección II se presenta una breve revisión de la literatura económica sobre los modelos de sostenibilidad fiscal. En la sección III se revisa el indicador de balance cíclicamente ajustado, clave en la articulación de la política fiscal chilena y, por ende, la base de la evaluación de sostenibilidad fiscal. En la primera parte de la sección IV se discuten los índices seleccionados de vulnerabilidad y sostenibilidad fiscal para el período 1990-2016, mientras que en la segunda se aplica el modelo dinámico propuesto. Finalmente, en la sección $V$ se presentan los comentarios y recomendaciones finales.

\section{Revisión de la literatura}

Los modelos de sostenibilidad fiscal pueden dividirse en tres grandes categorías. Primero se encuentran los de largo plazo, basados en el cálculo de un nivel de deuda sostenible en un estado estacionario de las finanzas públicas y con información futura sin incertidumbre. Entre estos se encuentran los indicadores de Blanchard (1990) y Buiter (1985). El segundo conjunto de modelos reconoce los cambios intertemporales que pueden sufrir las restricciones presupuestarias del gobierno, por lo que exploran las propiedades de series de tiempo para el balance fiscal y si estas cumplen con las condiciones suficientes para mantener las finanzas públicas en una senda sustentable (condición no Ponzi). Entre estos modelos están los desarrollados por Hamilton y Flavin (1986), Chalk y Hemming (2000) y Bohn (1998 y 2005). Este último trabajo, cuyo modelo utiliza también el Fondo Monetario Internacional (FMI, 2003), realiza una regresión del balance primario contra la deuda neta, con el propósito de encontrar evidencia de que la razón deuda/PIB posee reversión a la media. Por último, la tercera categoría introduce incertidumbre en variables tanto por el lado de los ingresos (en especial para economías que dependen de la venta de productos básicos) como en el mercado financiero. Algunos autores que han explorado esta categoría de modelos son Calvo, Izquierdo y Talvi (2003), quienes 
evalúan cambios en los precios relativos para la economía argentina, Barnhill y Kopits (2003), que incorporan incertidumbre con el método Value at Risk (VaR) y Mendoza y Oviedo (2006), quienes realizan un modelo de equilibrio general dinámico y estocástico, calculando el límite natural de la deuda para México. Mendoza y Oviedo (2009) presentan una versión más básica de este modelo, que incorpora incertidumbre por el lado de los ingresos fiscales y calcula el límite natural de deuda para cuatro países latinoamericanos.

Dentro de este último grupo también se encuentra el modelo del FMI para países en desarrollo (FMI, 2002, 2003 y 2010), que incorpora supuestos sobre las variables de corto plazo para evaluar su impacto en la sostenibilidad fiscal. En lo que se refiere a mediciones empíricas formales, que analizan específicamente el caso chileno, existen al menos dos trabajos que siguen una línea similar al modelo del FMI. Vergara (2002), quien estima el balance primario consistente con una razón de deuda pública neta a PIB del 20\% para distintas tasas de crecimiento y tasas de interés reales de largo plazo, y Crispi y Vega (2003), quienes evalúan dinámicamente la sostenibilidad de la política fiscal chilena, para lo cual utilizan una metodología de proyección de la deuda neta del gobierno central y analizan el efecto de la aplicación de la regla de balance cíclicamente ajustado sobre la trayectoria de la razón deuda neta a PIB para tres escenarios alternativos: un escenario base, un escenario base cíclico y un escenario histórico. El presente ejercicio se enmarca dentro de este tipo de análisis y contribuye a dicha literatura desarrollando un modelo dinámico de sostenibilidad, actualizado de acuerdo a las principales directrices de política fiscal vigente en Chile. Como aspecto novedoso se puede destacar que este trabajo sería uno de los primeros en incorporar la dinámica del FRP, que por mandato legal hasta 2016 puede considerarse como un componente importante de deuda, ya que hasta dicho año solo contemplaba una regla de acumulación. Además, se modela la regla de balance cíclicamente ajustado de acuerdo a su última actualización metodológica (DIPRES, 2017a).

\section{La regla de balance cíclicamente ajustado}

Desde 2001, la política fiscal chilena se ha guiado por una regla basada en un balance cíclicamente ajustado (BCA), conocido en la literatura como balance estructural del gobierno central total. Esta política tiene una mirada de mediano plazo, a diferencia del análisis tradicional basado principalmente en el balance efectivo, que da cuenta de una situación coyuntural.

La política de BCA consiste, a grandes rasgos, en estimar los ingresos del gobierno central ajustándolos por el ciclo económico y, por ende, autorizar un gasto público consistente con dichos ingresos. Así se busca eliminar el comportamiento tradicionalmente procíclico de la política fiscal ${ }^{4}$. Esto se traduce en ahorros de tiempos de expansión de la actividad económica, cuando se reciben ingresos mayores por causa del ciclo, para poder gastarlos ya sea cuando la economía se contrae y los ingresos fiscales caen, o cuando se enfrentan coyunturas que hacen que las necesidades de gasto aumenten.

La aplicación de la regla de BCA y su institucionalidad han sido consideradas exitosas y clave en el buen desempeño fiscal chileno, generando un apoyo transversal de amplios sectores de la sociedad. A nivel internacional se ha convertido en un referente de responsabilidad fiscal ${ }^{5}$. El Fondo Monetario Internacional (Dabán, 2011) define la regla como la "piedra angular del buen comportamiento fiscal de Chile".

Entre las bondades de la política de BCA chilena cabe mencionar que ha contribuido principalmente a: reducir la volatilidad fiscal y macroeconómica, aumentar el ahorro público durante

\footnotetext{
4 Véanse Gavin y otros (1996), Alberola y Montero (2007) e llzetzki y Végh (2008).

5 Para más detalles véanse BID (2008), FMI (2010) y OCDE (2010). Véase también Frankel (2011).
} 
los ciclos económicos expansivos, reducir las percepciones de riesgo sobre la economía chilena, reducir la volatilidad de la tasa de interés y ayudar a sostener un tipo de cambio real más competitivo y menos volátil, lo que ha permitido resguardar la competitividad del sector exportador, a pesar del pronunciado ciclo alcista en el precio del cobre observado a inicios de esta década 6 .

La política de BCA, en sus casi 20 años de implementación, ha estado sujeta a revisiones y cambios en su institucionalidad, en la meta de política, así como en el concepto y manera de medir el ciclo.

La política actual se sustenta en tres pilares (Larraín y otros, 2011):

1) Un indicador de BCA, que corresponde al conjunto de ecuaciones y variables que se necesitan para calcular un balance sin los efectos del ciclo económico.

2) Una meta de política, cuya definición es independiente de la medición del indicador de BCA, que establece el patrón de acumulación de pasivos netos libres del ciclo económico.

3) Un marco institucional y de transparencia, que apoya el buen funcionamiento de la regla.

De estos tres elementos, a continuación se procede a revisar únicamente las características del indicador de balance cíclicamente ajustado, para su posterior incorporación en el modelo de sostenibilidad.

En lo fundamental, el indicador de balance cíclicamente ajustado (BCA) consiste en definir un nivel de gasto coherente con un ingreso libre de fluctuaciones cíclicas del PIB y de los precios del cobre, sobre las cuales la autoridad no tiene control directo. El criterio subyacente es contar con un balance que permita la plena operación de los estabilizadores automáticos. Cualquier otro tipo de fluctuación de los ingresos no se ajusta, a fin de evitar distintas interpretaciones sobre qué es y qué no es permanente o transitorio e impedir acciones discrecionales de la autoridad.

En términos prácticos, esto supone no realizar correcciones a los ingresos asociados a medidas tributarias transitorias o a operaciones que hagan variar los ingresos por una vez. Se exceptúan las medidas tributarias de reversión automática - que se entienden como aquellas cuyos efectos en los ingresos fiscales son únicamente de caja, es decir, aquellas que solo implican adelantos o retrasos en el pago de impuestos-, las que sí están sujetas a correcciones. Quedan sin ajuste aquellas variaciones asociadas a eventos no relacionados con el ciclo y que como tales pudieran generar ciertos niveles de volatilidad en los ingresos cíclicamente ajustados (por ejemplo, variaciones del tipo de cambio o la inflación).

La cobertura institucional aplicada en la construcción del indicador es la del balance global del gobierno central total, por ser este el agregado sobre el cual se formula y ejecuta la política fiscal. Esta cobertura excluye a las empresas públicas, municipalidades y universidades estatales. Sin embargo, se debe resaltar que gran parte de la influencia de la política fiscal ejercida a través de estas instituciones está representada por las transferencias desde o hacia estos organismos, las que están reconocidas dentro del presupuesto del gobierno central.

Para la estimación del balance estructural se resta de los ingresos efectivos totales la sumatoria de los ajustes cíclicos de las distintas partidas de ingresos. En particular se aplica la siguiente fórmula:

$$
\begin{gathered}
B C A_{t}=B E f_{t}-A C_{t} \\
A C_{t}=I T N M_{t}^{c}+I C S_{t}^{c}+I T M_{t}^{c}+I C C_{t}^{c}
\end{gathered}
$$

\footnotetext{
6 Véanse por ejemplo Costa y Lagos (2001), Larraín y Parro (2006), Le Fort (2006), Rodríguez, Tokman y Vega (2006), Kumhof y Laxton (2009), Ffrench-Davis (2010), Chan-Lau y otros (2010), Marcel (2010), OCDE (2010), Schmidt-Hebbel (2010), Ter-Minassian (2010), Velasco y otros (2010), Frankel (2011), Tapia (2015) y Caputo y Valdés (2016).

7 Véase también Corbo y otros (2011).
} 
$B C A_{t}$ corresponde al balance cíclicamente ajustado en el período $t, B E f_{t}$ al balance efectivo en el período $t$ y $A C_{t}$ es el ajuste cíclico a los ingresos en el período $t$, que corresponde a la suma de los componentes cíclicos expresados por el superíndice "c" de las partidas: 1) tributación no minera (ITNM), 2) cotizaciones previsionales de salud (ICS), 3) tributación de la gran minería privada (ITM) y 4) traspasos de CODELCO por cobre (IC).

La partida de ingresos tributarios no mineros, así como el ajuste por cotizaciones previsionales de salud, se realiza según la metodología estándar utilizada por organismos internacionales, consistente en multiplicar los ingresos efectivos por la brecha del producto elevada a una elasticidad ${ }^{8}$. Por su parte, el ajuste correspondiente a la tributación de la gran minería privada y de los traspasos de CODELCO se ejecuta tomando en cuenta la diferencia entre el precio de referencia del cobre y el precio efectivo.

No se realizan ajustes cíclicos por los intereses de los activos o pasivos financieros y los restantes ingresos fiscales tampoco se encuentran sujetos a ajustes, al estar débilmente relacionados con el ciclo del producto. Asimismo, al no existir componentes del gasto que respondan de manera automática al ciclo económico, no se hace necesario ajustar el gasto. En DIPRES (2017a) puede encontrarse una revisión en detalle de la metodología de cálculo del indicador de BCA.

\section{Sostenibilidad fiscal de la economía chilena}

\section{Indicadores estáticos de vulnerabilidad y sostenibilidad fiscal}

A continuación se aborda el cálculo ex post de algunos indicadores seleccionados de vulnerabilidad y sostenibilidad fiscal adaptados a la realidad chilena?

Cabe destacar que la cobertura de gobierno utilizada para la medición de deuda que se presenta a continuación es el gobierno central presupuestario ${ }^{10}$. Esto implica excluir del análisis los pasivos y activos del Banco Central de Chile, de empresas públicas, universidades y municipalidades. Además, en la medición de los pasivos brutos del gobierno central presupuestario no se incluye el acervo de bonos de reconocimiento no liquidados, que constituyen un pasivo con los afiliados del antiguo sistema de pensiones que se cambiaron a las administradoras de fondos de pensiones (AFP). En efecto, dado que el acervo de bonos de reconocimiento se considera una obligación de naturaleza diferente de la considerada según el concepto tradicional de deuda del gobierno central, y a su vez de acuerdo a las prácticas a nivel internacional, estas estadísticas se presentan de manera complementaria y no son típicamente consolidadas con las estadísticas sobre deuda del gobierno central.

En el gráfico 2 se presenta la evolución de las finanzas del gobierno central como porcentaje del PIB desde 1990 hasta 2016, que en primera instancia dan origen a la situación de endeudamiento del gobierno y por lo tanto impactan directamente en los niveles de sostenibilidad fiscal. Se puede observar que, antes de la aplicación del BCA, el balance global ya mostraba valores positivos. Los años deficitarios, en general, se asocian con turbulencias en la economía, siendo de particular interés el año 2009, que acusa el mayor déficit de todo el período analizado (marcado por la crisis financiera internacional y por el plan de estímulo fiscal). Resalta, además, la tendencia a un déficit creciente que se observa marginalmente desde 2013 , con un $0,6 \%$, pero que es mucho más marcado durante los últimos tres años con un promedio de 2,1\%.

\footnotetext{
8 Véanse Giorno y otros (1995), Hagemann (1999) y Larch y Turrini (2009).

9 La fórmula de cálculo de estos indicadores se presenta en el anexo A1.

${ }^{10}$ Para un análisis que considera tanto el gobierno central como las empresas públicas y el banco central, véase Vergara (2002).
} 
Gráfico 2

Ingresos, gastos y balance del gobierno central, 1990-2016

(En porcentajes del PIB)

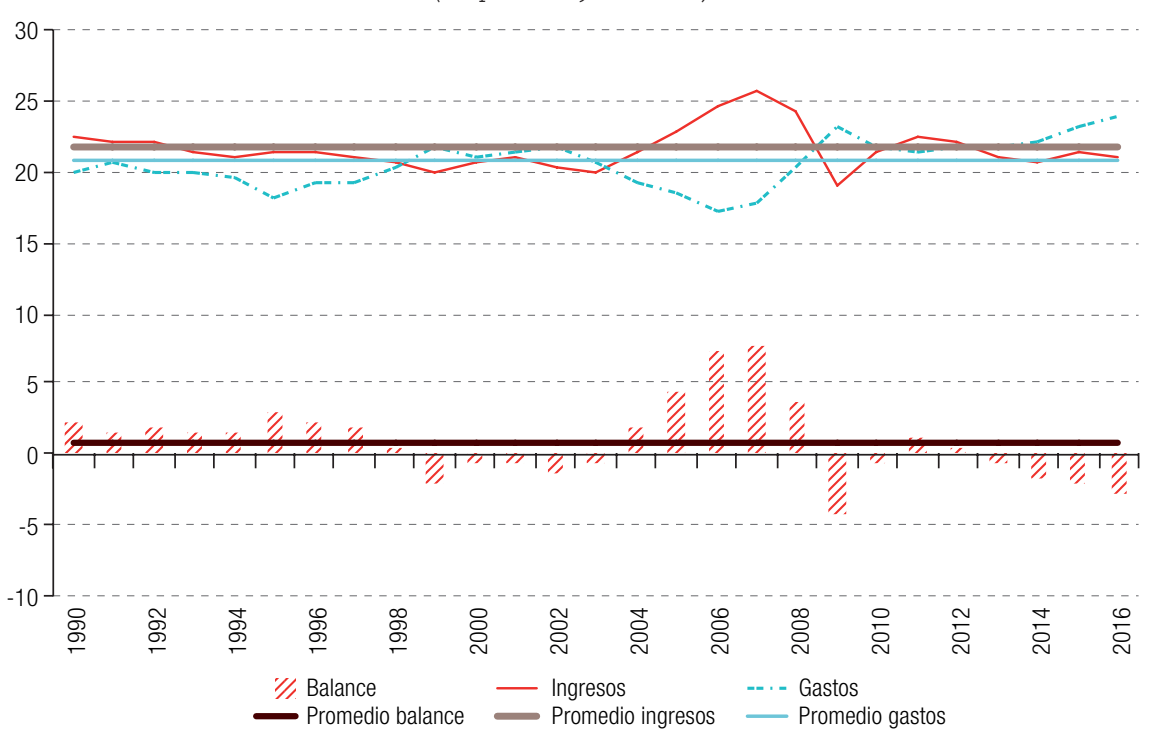

Fuente: Elaboración propia, sobre la base de información de la Dirección de Presupuestos (DIPRES).

A continuación, el cuadro 1 muestra la operación de los indicadores de sostenibilidad fiscal y permite además visualizar el impacto que tuvieron la crisis financiera internacional de 2008-2009 y el terremoto y tsunami de febrero de 2010 en la posición fiscal y sostenibilidad de la deuda del país. En la última hilera del cuadro se presenta el valor crítico o rango deseable de cada indicador, para tener una referencia de comparación con cada uno de los valores obtenidos anualmente. Se utilizaron diversos criterios para obtener estos valores o rangos. En los casos en que se encontraron valores de referencia publicados por el FMl, esos fueron los utilizados; en los casos en que no se encontraron estos valores, se asignó como valor crítico el que se desprende de la propia definición del indicador.

Cuadro 1

Chile: indicadores estáticos de vulnerabilidad y sostenibilidad fiscal, 1990-2016a (En porcentajes)

\begin{tabular}{lccccccc}
\hline Año & $\begin{array}{c}\text { Deuda bruta / } \\
\text { PIB }\end{array}$ & $\begin{array}{c}\text { Deuda neta / } \\
\text { PIB }\end{array}$ & $\begin{array}{c}\text { Servicio de } \\
\text { la deuda }\end{array}$ & $\begin{array}{c}\text { (IV) Prueba } \\
\text { ácida al servicio } \\
\text { de la deuda }\end{array}$ & $\begin{array}{c}\text { (V) Balance } \\
\text { primario } \\
\text { (metodología } \\
\text { FMI) }\end{array}$ & $\begin{array}{c}\text { (VI) Balance } \\
\text { primario } \\
\text { (metodología } \\
\text { DIPRES) }\end{array}$ & $\begin{array}{c}\text { (VII) Balance } \\
\text { primario } \\
\text { estructural }\end{array}$ \\
\hline 1990 & 44,0 & 29,4 & 23,5 & 29,7 & 5,4 & 5,4 \\
\hline 1991 & 38,0 & 22,9 & 24,9 & 27,9 & 4,5 & 4,6 \\
\hline 1992 & 31,2 & 15,1 & 19,5 & 21,4 & 4,4 & 4,5 \\
\hline 1993 & 28,6 & 13,6 & 20,7 & 21,6 & 3,5 & 3,6 \\
\hline 1994 & 23,0 & 8,2 & 15,6 & 16,8 & 3,4 & 3,4 \\
\hline 1995 & 17,5 & 4,0 & 21,6 & 24,6 & 4,6 & 4,7 & \\
\hline 1996 & 14,8 & 1,5 & 16,7 & 18,1 & 3,5 & 3,5 & \\
\hline 1997 & 12,9 & $-0,4$ & 11,8 & 12,9 & 3,2 & 3,2 & \\
\hline 1998 & 12,2 & $-0,3$ & 12,8 & 13,2 & 1,6 & 1,6 & $-0,9$ \\
\hline 2000 & 13,3 & 1,6 & 12,0 & 12,3 & $-0,8$ & 0,5 \\
\hline 2001 & 13,3 & 3,1 & 8,5 & 9,0 & 0,5 & 0,6 \\
\hline 2002 & 14,4 & 5,6 & 10,0 & 10,4 & 0,6 & 2,3 \\
\hline 2003 & 15,2 & 7,7 & 13,6 & 14,0 & $-0,1$ & $-0,1$ & 1,9 \\
\hline 2004 & 12,6 & 6,5 & 12,2 & 12,8 & 0,7 & 0,6 \\
\hline
\end{tabular}


Cuadro 1 (conclusión)

\begin{tabular}{|c|c|c|c|c|c|c|c|}
\hline Año & $\begin{array}{c}\text { Deuda bruta / } \\
\text { PIB }\end{array}$ & $\begin{array}{c}\text { Deuda neta / } \\
\text { PIB }\end{array}$ & $\begin{array}{l}\text { Servicio de } \\
\text { la deuda }\end{array}$ & $\begin{array}{l}\text { (IV) Prueba } \\
\text { ácida al servicio } \\
\text { de la deuda }\end{array}$ & $\begin{array}{l}\text { (V) Balance } \\
\text { primario } \\
\text { (metodología } \\
\text { FMl) }\end{array}$ & $\begin{array}{l}\text { (VI) Balance } \\
\text { primario } \\
\text { (metodología } \\
\text { DIPRES) }\end{array}$ & $\begin{array}{l}\text { (VII) Balance } \\
\text { primario } \\
\text { estructural }\end{array}$ \\
\hline 2005 & 7,0 & $-0,1$ & 15,2 & 19,4 & 5,2 & 5,0 & 1,8 \\
\hline 2006 & 5,0 & $-6,6$ & 8,0 & 12,3 & 8,0 & 7,7 & 1,8 \\
\hline 2007 & 3,9 & $-13,0$ & 6,7 & 9,9 & 8,3 & 7,8 & 1,1 \\
\hline 2008 & 4,9 & $-19,3$ & 6,3 & 8,2 & 4,4 & 3,7 & 2,9 \\
\hline 2009 & 5,8 & $-10,5$ & 6,3 & 7,3 & $-3,8$ & $-4,3$ & $-2,9$ \\
\hline 2010 & 8,6 & $-7,0$ & 4,1 & 5,2 & 0,0 & $-0,2$ & $-1,5$ \\
\hline 2011 & 11,1 & $-8,6$ & 4,7 & 5,7 & 1,8 & 1,5 & $-0,5$ \\
\hline 2012 & 11,9 & $-6,8$ & 6,2 & 7,2 & 1,1 & 0,8 & $-0,6$ \\
\hline 2013 & 12,7 & $-5,6$ & 6,9 & 7,7 & 0,0 & $-0,3$ & $-1,3$ \\
\hline 2014 & 14,9 & $-4,3$ & 8,0 & 8,8 & $-1,0$ & $-1,2$ & $-1,5$ \\
\hline 2015 & 17,4 & $-3,5$ & 8,6 & 9,1 & $-1,5$ & $-1,7$ & $-1,0$ \\
\hline 2016 & 21,3 & 1,0 & 42,3 & 43,0 & $-2,0$ & $-2,3$ & $-1,0$ \\
\hline Promedio & 15,8 & 1,4 & 13,3 & 15,0 & 2,2 & 2,0 & 0,3 \\
\hline $\begin{array}{l}\text { Valor crítico o } \\
\text { rango deseable }\end{array}$ & 25-30 (FMI) & $42,8^{b}$ & 25-35 (FMI) & 25-35 (FMI) & Valor $=0$ & Valor $=0^{c}$ & Valor $=0$ \\
\hline
\end{tabular}

Fuente: Elaboración propia, sobre la base de información de la Dirección de Presupuestos (DIPRES) y el Banco Central de Chile.

a Para la medición de los indicadores la tasa de interés ha sido calculada como la tasa de interés nominal implícita tanto de los pasivos como de los activos.

b Promedio países de la OCDE (1993-2010), gobierno general.

c Al ser un indicador sostenible su promedio durante el período constituye un valor de referencia para cada año.

Las cifras muestran una tendencia decreciente de la deuda bruta desde 1990 hasta la crisis financiera de 2009, cuando esta tendencia se revierte y el endeudamiento neto como porcentaje del PIB de cada año vuelve a crecer. Estos resultados se reflejan a su vez en el comportamiento de la deuda neta como porcentaje del PIB. La deuda neta resulta de restarle a la deuda bruta los activos del gobierno central (Fondo de Estabilización Económica y Social, Fondo de Reserva de Pensiones y otros). Esta presenta una evolución sumamente descendente desde 1997 hasta 2008, atribuible tanto a reducciones de deuda bruta como a incrementos de los activos, que se deben en buena medida al importante componente cíclico de los ingresos del cobre, cuyo precio registró un marcado aumento hacia mediados de los años 2000, lo que en el marco de la regla fiscal permitió un gran incremento del ahorro. En 2009, en el marco de la política contracíclica financiada, en parte, con recursos del FEES, se observó una reversión de esta, aunque continuó siendo negativa. En la actual administración ha continuado el deterioro de las cifras fiscales, siendo 2016 el primer año en una década en el cual Chile ha dejado de ser un acreedor neto.

De conformidad con lo anterior, el servicio de la deuda también mostró una reducción significativa. En promedio simple, previo a la operación de la regla estos egresos significaron cerca del $18 \%$ de los ingresos corrientes del gobierno central y hasta el $20 \%$ de no considerar el cobre dentro de estos ingresos. Por su parte, entre 2000 y 2015 el servicio de la deuda cae a un promedio simple del $9 \%$ de los ingresos corrientes y del 10\% si no se consideran los aportes del cobre. Destaca 2016, donde se observa un abrupto crecimiento del servicio de la deuda que alcanza cerca de la mitad de los ingresos corrientes del gobierno central, valores que responden fundamentalmente a amortizaciones de la deuda realizadas en dicho año.

Respecto del balance primario según la metodología FMI, que descuenta de los gastos el pago de intereses, dada la evolución favorable de la deuda, tendió a igualarse al balance global, producto de la reducción de intereses como proporción del total de gastos. Destaca además que antes de 2014 este indicador presentó valores negativos en solo tres años, todos estos asociados a crisis financieras externas. A partir de 2014 las mayores presiones de gasto han llevado a valores negativos 
del indicador en tres años consecutivos, hecho inédito en las cuentas fiscales de las últimas dos décadas. Algo similar se observa en el caso del indicador de balance primario según la metodología de la Dirección de Presupuestos (DIPRES), que descuenta también los ingresos provenientes de los intereses de los activos financieros del gobierno central.

Al corregir los ingresos por el ciclo económico se observa un balance cíclicamente ajustado (BCA) primario positivo y relativamente estable hasta 2008. Este período se caracterizó por el mantenimiento de una meta de balance estructural del 1\% del PIB hasta 2007 y una meta del 0,5\% en 2008. En 2009, a raíz del paquete fiscal implementado, que repercutió en un déficit cíclicamente ajustado del 3,1\% del PIB, el BCA primario se tornó negativo. A partir de dicho año y con una meta de convergencia, este indicador presenta valores negativos.

En síntesis, se registra una mejora de los indicadores durante los primeros años de operación de la regla fiscal, cuando esta mantuvo una meta de un superávit estructural del 1\% del PIB. A partir de 2009 con la crisis financiera internacional, 2010 con el terremoto y tsunami y desde 2011 con las crecientes presiones al gasto fiscal, las cifras han tendido a deteriorarse, aunque se mantienen en mejores niveles que los observados a inicios de la década de 1990.

\section{La dinámica de la política fiscal y su sostenibilidad en el marco de la política de balance cíclicamente ajustado}

En esta sección se analiza la sostenibilidad fiscal en el marco de la regla fiscal en Chile desde un punto de vista dinámico.

\section{a) Antecedentes metodológicos}

La proyección de los balances futuros y, por ende, de la deuda futura es un elemento clave en el estudio de la sostenibilidad de las finanzas públicas. Según se ha observado en diversos países, los desequilibrios fiscales pueden derivar en pronunciadas crisis, con efectos que podrían perdurar por muchos años. Por ello, resulta necesario analizar tanto el acervo de deuda que mantiene un país como el flujo que se genera todos los años. El flujo, en este caso, está determinado por los déficits o superávits fiscales que presenten año a año los balances de gobierno.

Lo que se describirá a continuación es un modelo que analiza la senda de la deuda, teniendo en cuenta tanto el acervo como los flujos. El énfasis estará definido en las variables ex post ${ }^{11}$.

\section{i) Economía de los modelos de sostenibilidad}

La literatura sobre sostenibilidad fiscal tiene como base la siguiente ecuación, que muestra la relación intertemporal entre la deuda y el déficit operacional del gobierno en términos nominales:

$$
D_{t}=\left(1+i_{t}\right) D_{t-1}-B P_{t}-\Delta M_{t}
$$

\footnotetext{
${ }^{11}$ Así se simulan las variables macroeconómicas que determinan los ingresos y gastos del fisco y luego se analiza el movimiento de la deuda en el tiempo. Un análisis ex ante sería más riguroso, pero mucho menos sencillo: las variables que aquí se considerarán suelen ser endógenas e inciertas, es decir, se determinan por su movimiento en conjunto y no poseen una senda clara para el futuro. Un ejemplo documentado es la influencia del gasto público en el tipo de cambio (Arellano y Larraín, 1996). A pesar de esta simplificación, el álgebra ex post de los modelos de sostenibilidad sigue siendo una herramienta poderosa para identificar posibles riesgos futuros (Ley, 2010).
} 
Donde $D_{t}$ es el acervo de deuda acumulada al final del período $t, i_{t}$ es la tasa de interés nominal, $\Delta M_{t}$ es el aumento de la base monetaria al finalizar el período $t$ (señoreaje) y $B P_{t}$ es el balance primario ${ }^{12}$ en el período $t$. Para reflejar el movimiento de la deuda en un año, se resta $D_{t-1}$ en ambos lados de la ecuación, obteniendo:

$$
\begin{gathered}
D_{t}-D_{t-1}=\left(1+i_{t}\right) D_{t-1}-B P_{t}-\Delta M_{t}-D_{t-1} \\
\Delta D_{t}=i_{t} D_{t-1}-B P_{t}-\Delta M_{t}
\end{gathered}
$$

Finalmente, si se desea expresar la ley de movimiento de la deuda como porcentaje del PIB, la ecuación queda descrita de la forma siguiente:

$$
\Delta d_{t}=\frac{r_{t}-g_{t}}{1+g_{t}} d_{t-1}-b p_{t}-\Delta m_{t}
$$

Donde las variables en minúsculas denotan estar expresadas como porcentaje del PIB, $r_{t}$ es la tasa de interés real en el período $t$ y $g_{t}$ es la tasa de crecimiento real del producto en el período $t$. Si esta ecuación quiere expresarse en función del balance efectivo, se obtiene:

$$
\Delta d_{t}=\frac{1+i_{t}}{1+\gamma_{t}} \gamma_{t} d_{t-1}-\Delta m_{t}-b e f_{t}
$$

Donde $i_{t}$ es la tasa de interés nominal en el período $t, \pi_{t}$ es la inflación en el período $t$ y $\gamma_{t}$ es igual a la suma de $\pi_{t}+g_{t}+\pi_{t} g_{t}$.

En el caso chileno existen, además de estos aspectos, tres características que deben ser consideradas al momento de realizar un análisis de sostenibilidad fiscal: 1) la regla de balance cíclicamente ajustado, 2) la importancia de los ingresos del cobre y 3) la política de fondos soberanos que el país posee. En el siguiente apartado se analizan estas tres características y su incorporación en el cálculo de sostenibilidad fiscal.

\section{ii) Características de la economía chilena}

Siguiendo la regla fiscal chilena, el gasto de gobierno se fija según la meta de BCA, definida como porcentaje del producto para cada año. Esto implica que el resultado del balance primario estará determinado fundamentalmente por el ajuste cíclico y la meta de BCA del año.

Tal como se expuso en la ecuación (1), el BCA es igual al balance efectivo menos el ajuste cíclico de los ingresos. Así, es posible expresar:

$$
\begin{gathered}
B C A_{t}=B E f_{t}-A C_{t} \\
B E f_{t}=I T_{t}-G T_{t} \\
B C A_{t}=\left(I T_{t}-G T_{t}\right)-A C_{t} \\
B C A_{t}=\left(I T_{t}-G T_{t}\right)-\left(I T_{t}-I T_{t}^{*}\right) \\
B C A_{t}=I T_{t}^{*}-G T_{t}
\end{gathered}
$$

\footnotetext{
${ }^{12}$ Aunque en esta sección se restan solo los gastos por intereses, implícitamente se utiliza la definición de balance primario de DIPRES, ya que los intereses se calculan sobre la deuda neta, por lo que, tal como se menciona más adelante, puede que el gasto por intereses sea negativo, es decir, que los ingresos por intereses superen los intereses a pagar por la deuda bruta acumulada.
} 
Donde $B C A_{t}$ es el BCA, $B E f_{t}$ es el balance efectivo, $A C_{t}$ es el ajuste cíclico de los ingresos, $I T_{t}$ son los ingresos totales efectivos, $G T_{t}$ es el gasto total del gobierno y $I T_{t}^{*}$ son los ingresos cíclicamente ajustados.

Por otra parte, el ajuste cíclico a los ingresos se simplificará en este modelo, definiéndolo de la siguiente manera:

$$
\begin{gathered}
A C_{t}=I T N M_{t, i} \cdot\left(1-\left(\frac{Y_{t}^{*}}{Y_{t}}\right)^{\eta}\right)+\left(V C_{t} \cdot\left(P_{t}^{C}-P_{t}^{*}\right)+\right) \cdot e_{t} \cdot 2.204,62 \\
+\left(P_{t}^{B M L}-P_{t}^{*}\right) \cdot \tilde{\tau}_{t} \cdot Q_{t} \cdot e_{t} \cdot 2.204,62
\end{gathered}
$$

En este caso, se agrupan todos los ingresos tributarios no mineros (junto con las cotizaciones de salud) en el término $I T N M_{t}$ y se utiliza una elasticidad agregada promedio $\eta$. $Y^{*}$ representa el producto tendencial.

La fórmula de ajuste cíclico a los ingresos que provienen de la Corporación Nacional del Cobre (CODELCO) que se utiliza en este modelo es idéntica a la descrita en DIPRES (2017a), donde $P_{t}^{C}$ y $P_{t}^{*}$ son el precio del cobre efectivo y de referencia, respectivamente, $V F$ es la producción (venta) ${ }^{13}$ física de cobre y $e_{t}$ corresponde al tipo de cambio nominal del período.

El ajuste cíclico a la tributación de la gran minería privada también se simplifica. En vez de ajustar por separado los tres tipos de impuestos que estas empresas pagan, se utiliza una tasa promedio $\tau_{t}$ para representar la carga tributaria de las empresas mineras por su producción $Q$ en el año $t$. De todas formas, dicha tasa promedio se calcula ponderando las tasas de cada tipo de impuesto, es decir, las del impuesto específico, a la renta de primera categoría y adicional. Esta tasa, además de tomar en cuenta el efecto de las remesas en el impuesto adicional, debe considerar los cambios tributarios en el impuesto específico a la actividad minera ${ }^{14}$.

Por último, cabe recordar que la regla fiscal chilena fija el gasto de gobierno respecto a los ingresos cíclicamente ajustados y la meta de balance cíclicamente ajustado. Por lo tanto, si el resultado estructural está fijo por meta, el gasto se determina de la siguiente manera:

$$
G T_{t}=I T_{t}^{*}-\overline{B C A_{t}}
$$

Junto con lo anterior, parte importante de los ingresos del gobierno central total proviene de la venta de cobre. Sin embargo, el precio del cobre ha tenido un ciclo pronunciado a lo largo de la historia, lo que se ha visto reflejado en una recaudación fluctuante por parte del gobierno (Borensztein y otros, 2010). Por lo anterior, tanto los ingresos por concepto de traspasos de CODELCO como la recaudación tributaria de la gran minería privada son considerados, en el modelo, como un componente diferenciado del resto en los ingresos efectivos del gobierno y luego son ajustados por el ciclo del precio del cobre ${ }^{15}$.

Para efectos del modelo, los ingresos y gastos se descompondrán de la siguiente manera:

$$
\begin{gathered}
I T_{t}=I T N M_{t}+I T M_{t}+\text { Codelco }_{t}+i_{t}^{R} \cdot \text { FRP }_{t-1}+\text { resto }_{t} \\
G T_{t}=\text { GOtros }_{t}+i_{t} \cdot D_{t-1}+\text { GPrev }_{t}
\end{gathered}
$$

\footnotetext{
${ }_{13}^{13}$ Para la proyección de las ventas se considera como el mejor indicador la cantidad producida.

${ }^{14}$ Aunque el impuesto a la renta y el específico se pagan por la producción y ventas en $t$ - 1 , hacer depender $\tau$ de $Q_{t-1}$ no permitiría tomar en cuenta el impuesto adicional. Sin embargo, hacer depender todo de $t$ sí permite capturar el efecto de los impuestos a la renta y específico, mediante los pagos provisionales mensuales que estos pagan durante el año.

15 Sobre un eventual término de este recurso natural, no se prevé que ocurra sino hasta dentro de muchas décadas. Véase Borensztein y otros (2010).
} 
Donde $I T N M_{t}$ son los ingresos tributarios no mineros y cotizaciones de salud en el período $t$; $I T M_{t}$ son los ingresos tributarios mineros; Codelco son los ingresos por traspasos de CODELCO y $i^{R} F R P_{t-1}$ son los ingresos por intereses del FRP; todos los ingresos que no son función de las variables macroeconómicas explicitadas en este modelo se asumen exógenos y se agrupan en la variable resto.

Por su parte, el término $i_{t} D_{t-1}$ son los pagos de intereses de la deuda neta ${ }^{16}$ en el período $t$; GPrev $_{t}$ es el gasto previsional, que es exógeno y explicado por razones demográficas, y GOtros es el resto de los gastos, que es la variable de ajuste para que el gasto total sea igual a los ingresos cíclicamente ajustados menos la meta de balance estructural.

Los ingresos del gobierno están en función de las variables macro de la siguiente manera ${ }^{17}$ :

$$
\begin{gathered}
\operatorname{ITNM}_{t}=\operatorname{ITNM}_{t-1} \cdot\left(1+\frac{\Delta P}{P_{t-1}}\right)\left(\frac{\Delta Y}{Y_{t}}\right)^{n} \\
\operatorname{ITM}_{t}=\tilde{\tau}_{t} \cdot M g_{t}^{P} \cdot Q_{t} \cdot e_{t} \cdot 2.204,62 \\
\text { Codelco }=\left(M g_{t}^{C} \cdot V F_{t}\right) \cdot e_{t} \cdot 2.204,62 \\
M g_{t}^{P}=P_{t}^{B M L}-c_{t}^{P} \\
M g_{t}^{C}=P_{t}^{C}-c_{t}^{C}
\end{gathered}
$$

Donde $c^{P}$ y $c^{C}$ son los costos unitarios de la gran minería privada y CODELCO, respectivamente.

Por último, en septiembre de 2006 se promulgó la Ley №20.128 sobre Responsabilidad Fiscal, la que estableció, entre otras cosas, la exigencia de ahorrar activos en el Fondo de Reserva de Pensiones (FRP) y el Fondo de Estabilización Económica y Social (FEES). Al 31 de agosto de 2017, estos fondos han acumulado 10.155 millones de dólares y 14.769 millones de dólares, respectivamente. Una característica a resaltar de estos fondos son sus claros objetivos y reglas de acumulación ${ }^{18}$.

El FRP fue diseñado con el fin de cubrir los pasivos de carácter previsional del gobierno central. En particular, los giros anuales que se podían realizar del FRP fueron, hasta 2016, por un máximo equivalente a la rentabilidad que este generara. Después de 2016, el máximo giro equivaldrá a un tercio de la diferencia entre el gasto previsional del año $t$ y el gasto equivalente en 2008. Por su parte, la regla de acumulación del FRP sigue la siguiente fórmula:

$$
P_{t-1} Y_{t-1} \cdot \operatorname{mín}\left\{0,5 \% ; \operatorname{máx}\left(0,2 \% ; B E f_{t-1} / P_{t-1} Y_{t-1}\right)\right\}
$$

Donde $P_{t-1} Y_{t-1}$ y $B E f_{t-1}$ son el PIB nominal del período pasado y el balance efectivo del período pasado, respectivamente. Esto indica que el aporte mínimo al fondo será del 0,2\% del PIB del año anterior y el máximo equivaldrá al 0,5\% del PIB del año anterior, dependiendo del balance efectivo obtenido. Como se aprecia, la existencia del FRP, los intereses que genere y sus reglas de acumulación y desacumulación son parte central de lo que será la discusión de sostenibilidad. Además, en el modelo, en un primer período (hasta 2016) los flujos de aportes periódicos a este fondo (que como

\footnotetext{
${ }^{16}$ Si la deuda neta es negativa esta expresión también lo será, lo que es equivalente a tener mayores ingresos por intereses que gastos por intereses.

17 Al respecto, véase el capítulo VIII de DIPRES (2013), cuyo estudio aplica fórmulas similares para la proyección de ingresos fiscales de largo plazo. También se pueden revisar los anexos en Crispi y Vega (2003) y Larraín y otros (2011) para la explicación de estas formas funcionales.

18 Para una revisión de los objetivos que puedan tener los fondos soberanos y sus diferentes tipos, véanse FMl (2008) y Kunzel y otros (2010). Para ver el marco legal y el funcionamiento de los fondos chilenos, véase Contreras y otros (2008).
} 
acervo es un activo) son tratados como un componente más de deuda, al contemplar hasta esa fecha únicamente una regla de acumulación, para después de 2016 poder optar a convertirse en otra fuente de recursos.

Por su parte, el FEES está diseñado para actuar como fondo de estabilización del gasto público, en el contexto de la política de BCA. Dado que el gasto se fija de acuerdo a los criterios de mediano plazo que arroja la regla fiscal chilena, el resultado del balance efectivo puede ser positivo o negativo, según la parte en que se encuentre el ciclo. El FEES acumula recursos del superávit efectivo que exceda el $0,5 \%$ del PIB del año y sirve como herramienta adicional al endeudamiento para financiar déficits. Tal como aparece en Walker (2011), este es un fondo cuyo valor esperado en el largo plazo es cero, además de no tener reglas de desacumulación preestablecidas, como sí ocurre con el FRP. El gobierno, ante un escenario de déficit, puede optar indistintamente entre utilizar recursos del FEES, vender otros activos o incurrir en endeudamiento interno o endeudamiento externo. Es una decisión discrecional de la autoridad y suele asociarse a las condiciones macroeconómicas que imperen en el momento. Por lo tanto, en un modelo simplificado como el que se expondrá en la siguiente sección, el efecto de incluir al FEES como elemento de activos dentro de la deuda neta externa no es relevante para el objetivo de este análisis. Es decir, el FEES no se modelará como una variable independiente a la deuda neta, como sí se hará con el FRP.

\section{iii) Modelo de sostenibilidad fiscal en el contexto de la economía chilena}

Siguiendo a Talvi y Végh (2000), quienes ponen énfasis en el déficit fiscal más que en el déficit operacional del gobierno, el nivel de cobertura quedará restringido al gobierno central total. Con esto, se prescinde de la variable de creación de dinero, $\Delta M_{t}$, de las ecuaciones.

Para determinar la necesidad de financiamiento del gobierno, es decir, si debe generar nueva deuda o puede amortizar la ya existente, el cálculo ex post se lleva a cabo de acuerdo con el flujo del diagrama 1:

\section{Diagrama 1}

Flujograma de cálculo del modelo

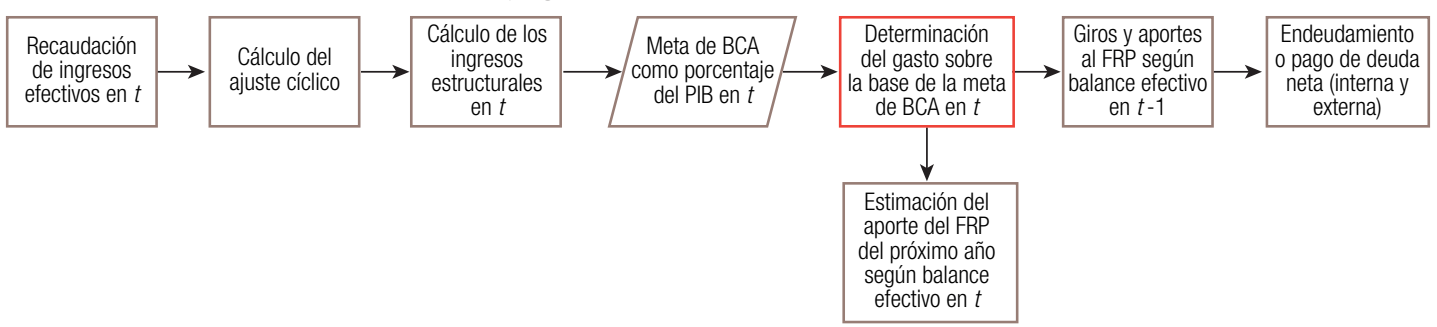

Fuente: Elaboración propia.

Como se mencionó, para efectos de este análisis, los ingresos del gobierno central se separan en cinco categorías: 1) recaudación tributaria no minera, 2) recaudación tributaria minera, 3) traspasos de CODELCO, 4) intereses que genera el FRP y 5) el resto de ingresos. De estas, las tres primeras se ajustan por ciclo del PIB o del precio del cobre, según corresponda. Con los ingresos cíclicamente ajustados y la meta, se determina el gasto del gobierno, que considera tres categorías de erogaciones: 1) el gasto previsional, 2) el pago de intereses de la deuda ${ }^{19}$ y 3) el resto de los gastos. Estos movimientos pueden catalogarse, según el Manual de Estadísticas del FMI (2001), como operaciones "sobre la línea". Por su parte, entre las operaciones "bajo la línea" (de financiamiento)

\footnotetext{
${ }^{19}$ Dado que se trabaja con deuda neta, puede que el gasto por intereses resulte ser negativo, es decir, que los intereses que generan los activos superen a los intereses de los pasivos.
} 
están los aportes y giros al FRP junto con la generación (o amortización) de deuda. En el caso de que el balance efectivo sea positivo, este sirve como fuente de recursos; en caso contrario, se suma a los usos de los recursos obtenidos mediante deuda 20 .

Especificados los movimientos que ocurren "sobre" y "bajo la línea", la ecuación para determinar el acervo y el flujo de deuda tiene la siguiente forma ${ }^{21}$ :

$$
\begin{gathered}
D_{t}=\left(1+i_{t}\right) \cdot D_{t-1}+\Delta F R P_{t}-B P_{t} \\
D_{t}=\left(1+i_{t}\right) \cdot D_{t-1}-B P_{t}^{\prime} \text { con } B P_{t}^{\prime}=B P_{t}-\Delta F R P_{t} \\
\Delta D_{t}=i_{t} D_{t-1}-B P_{t}^{\prime}=-B E f_{t}^{\prime}
\end{gathered}
$$

Donde $\Delta F R P_{t}$ es la variación del FRP en el período $t$. Se aprecia entonces que el FRP es separado del componente de deuda neta en dólares. Así, la variación del acervo de deuda para un año como porcentaje del PIB viene dada por la ecuación:

$$
\Delta d_{t}=\frac{r_{t}-g_{t}}{1+g_{t}} d_{t-1}+\frac{\Delta F R P_{t}}{P_{t} Y_{t}}-b p_{t}
$$

Así, realizando una descomposición similar a la ecuación (5) y dado que el balance efectivo es igual al BCA más el ajuste cíclico de los ingresos, es posible relacionar el movimiento de la deuda como porcentaje del PIB con la meta de BCA de la siguiente forma:

$$
\Delta d_{t}=-\frac{1-i_{t}}{1+\gamma_{t}} \gamma_{t} d_{t-1}+\frac{\Delta F R P_{t}}{P_{t} Y_{t}}-\left(\overline{b c a_{t}}-a c_{t}\right)
$$

Donde $\overline{b c a_{t}}$ es la meta de BCA como porcentaje del PIB en el año $t$ y $a c_{t}$ es el ajuste cíclico como porcentaje del PIB en el año $t$.

\section{b) Proyecciones de deuda neta a 2025}

A fin de ejemplificar el funcionamiento del modelo dinámico desarrollado en las secciones anteriores, a continuación se simula la senda de deuda neta del gobierno central de Chile hacia 2025. Cabe destacar que los valores iniciales de las principales variables utilizadas en este ejercicio son consistentes con el presupuesto 2018 y la proyección financiera del sector público para el período 2019-2021, entregada por el Ministerio de Hacienda en el Informe de Finanzas Públicas (DIPRES, 2017b).

A continuación se describen los dos escenarios macroeconómicos alternativos utilizados en el análisis22:

i) Escenario de tendencia: se considera como posición inicial lo proyectado en el presupuesto 2018 y la proyección financiera del sector público para el período 2019-2021 y una convergencia a sus valores de tendencia. Entre otras cosas, se asume que:

\footnotetext{
20 Sobre los términos "Fuentes" y "Usos", véase información proporcionada en la nota a pie de página del Cuadro I.17 del informe de Evaluación de la Gestión Financiera 2017 (DIPRES, 2017c).

${ }^{21}$ Entre los pasivos con los que cuenta el fisco, que se registran "bajo la línea", se cuentan los bonos de reconocimiento (Arenas y otros, 2009). Se ha decidido, tal como lo aborda Vergara (2002), contabilizar dentro de la deuda solo los bonos de reconocimiento ya emitidos. Así el ejercicio de sostenibilidad no pierde validez, ya que en términos de solvencia no hay diferencias por parte del gobierno entre una cesación de pagos en un bono de reconocimiento u otro bono.

22 El detalle de las variables macroeconómicas utilizadas en la simulación de cada escenario se presentan en el anexo A3. Para un mayor análisis del modelo utilizado, véase Bravo y otros (2014).
} 
a. La brecha entre el PIB efectivo y el tendencial se mantiene en cero desde 2022.

b. El precio del cobre alcanzaría en 2021 su valor de referencia de 2,77 dólares la libra.

c. La meta de balance estructural converge de un -1,2\% del PIB en 2019 a un balance equilibrado en 2024, a una razón anual de un 0,25\% del PIB.

ii) Escenario adverso: este escenario alternativo considera un crecimiento muy acotado para el producto durante 2018, de solo un 0,5\% real anual. El origen de esta fuerte desaceleración es externo, ya que asume una caída del precio del cobre a 1,5 dólares la libra, con una depreciación del peso y una respuesta rápida de las economías tanto internas como externas con una reducción de las tasas de interés.

Los rezagos de la desaceleración continúan en 2019 y el PIB comienza a crecer a tasas sobre un 2\% en 2021, convergiendo hacia una brecha PIB del 1,8\% en 2025.

Cabe destacar que, en este escenario, no se simulan variaciones en el valor de los activos financieros, por lo que el impacto sobre el endeudamiento neto se encuentra dado únicamente por el impulso fiscal simulado. Finalmente, este escenario simula los resultados de distintas metas de balance estructural para afrontar la desaceleración y el efecto en la deuda neta de cada una de estas alternativas.

Por último, dado lo observado con el gasto previsional durante los últimos años, en este ejercicio para ambos escenarios se prescinde del FRP como fuente de ingresos en el largo plazo, con un gasto previsional creciendo a tasas reales del orden del 1,5\%. Es decir, no se utilizan ni los intereses que este fondo genera ni las eventuales desacumulaciones que pueden realizarse a partir de 2016.

\section{i) Resultados}

A continuación, en el gráfico 3 se expone en el escenario de tendencia el movimiento de la deuda neta por sí sola e incluyendo el FRP.

Gráfico 3

Escenario de tendencia

A. Deuda neta, sin incluir el Fondo de Reserva de Pensiones (FRP)

(en porcentajes y millones de pesos)

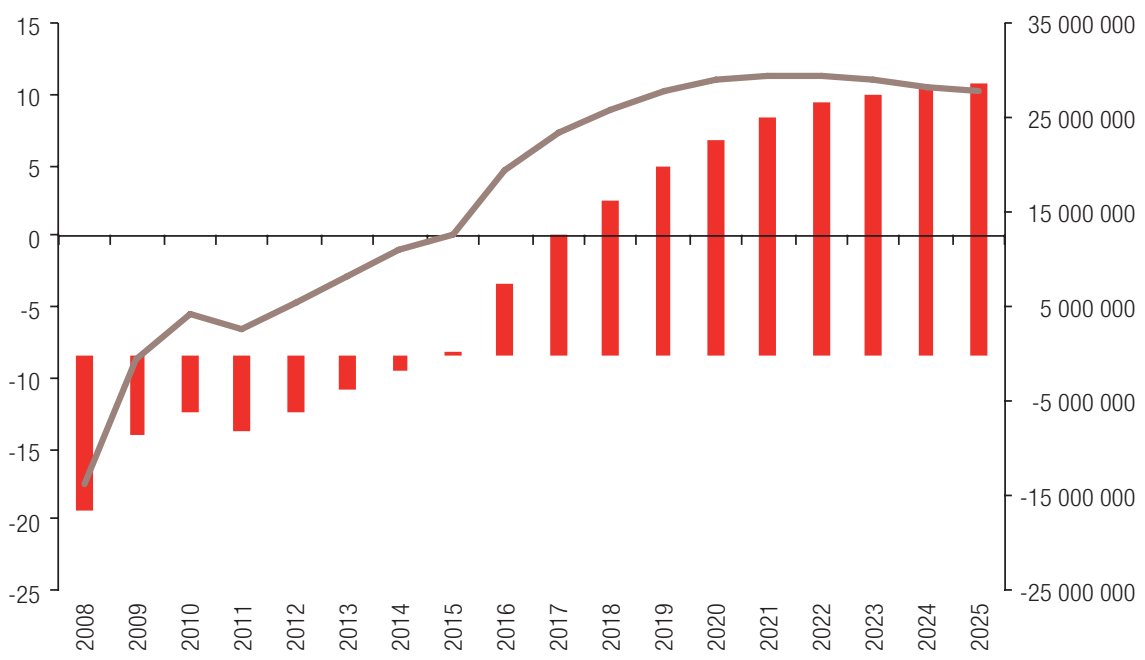

En millones de pesos _ Como porcentaje del PIB 
B. Deuda neta, incluido el Fondo de Reserva de Pensiones (FRP) (en porcentajes y millones de pesos)

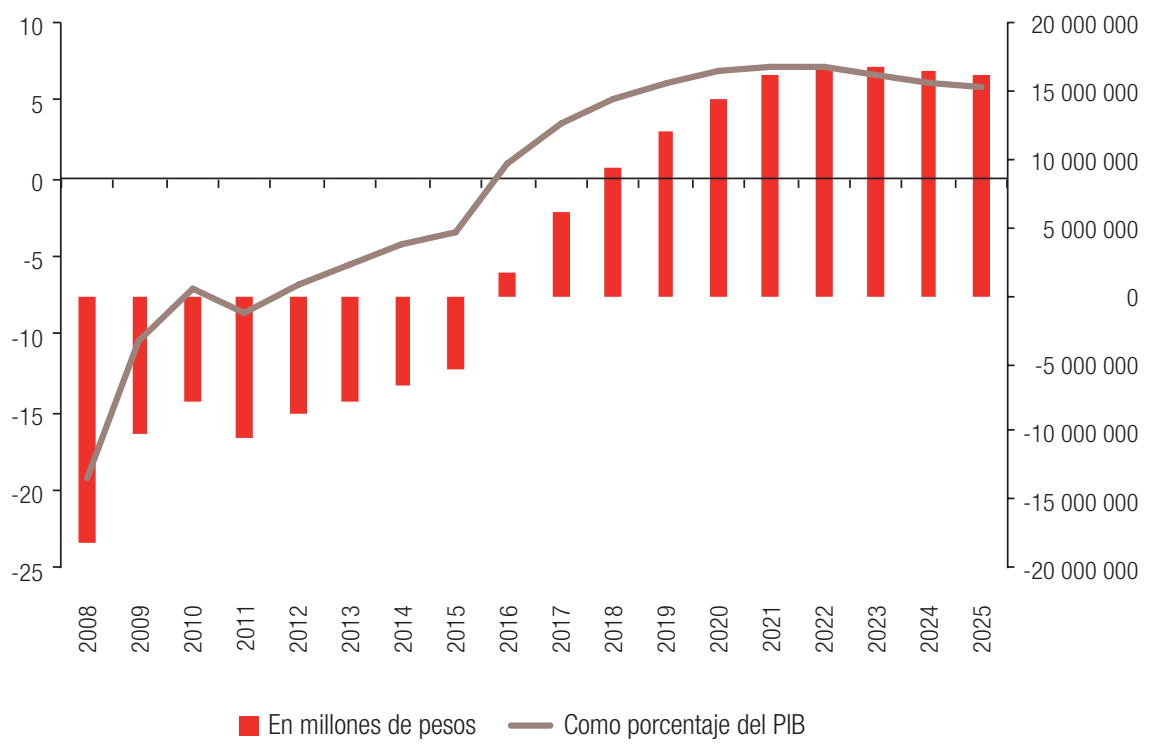

Fuente: Elaboración propia.

Como se aprecia en el gráfico 3, en la situación inicial del gobierno central el nivel de deuda neta es cercano al equilibrio, es decir, en 2016 la deuda bruta supera ligeramente el nivel de activos. Esto ocurre incluso considerando dentro de la deuda neta solo al FEES y a los otros activos del gobierno central.

La proyección considera un paulatino aumento de la deuda neta, que se estabiliza como porcentaje del PIB a partir de 2021. Dado el escenario asumido, donde la brecha del producto se cierra en 2022, y con las diferencias entre el balance efectivo y el cíclicamente ajustado explicadas por lo que ocurre con el mercado minero, que a su vez va cerrando sus diferencias entre los precios efectivos y de tendencia, los déficits cíclicamente ajustados también implican déficits efectivos, lo que significa acumular deuda o liquidar activos (ya sea del FEES o de los otros activos). A continuación, en el gráfico 4 se ilustra el efecto en el gasto del gobierno de ceñirse a la meta de balance cíclicamente ajustado, en lo que respecta al crecimiento real del gasto y a su medición como porcentaje del PIB. Se observa una reducción paulatina del gasto como porcentaje del PIB a partir de 2018, lo que está de acuerdo con una meta de balance cíclicamente ajustado que tiende al equilibrio. En este escenario las variaciones reales del gasto se encontrarían en torno a un 2,5\% real anual.

En el cuadro 2 se aplica el cálculo de los indicadores II, VI y VII, que figuran en el cuadro $1^{23}$, a la simulación.

\footnotetext{
${ }^{23}$ No se simulan todos los indicadores presentados en las secciones previas debido a que el modelo descrito no permite estimar todas las variables. Por ejemplo, si bien se presentaron previamente el balance primario con metodología FMl y DIPRES, en esta parte del documento se considera solamente el indicador construido por DIPRES, dado que el modelo solo separa los ingresos por intereses del FRP.
} 


\section{Gráfico 4}

Gasto total: crecimiento real y PIB en escenario de tendencia

(En porcentajes)

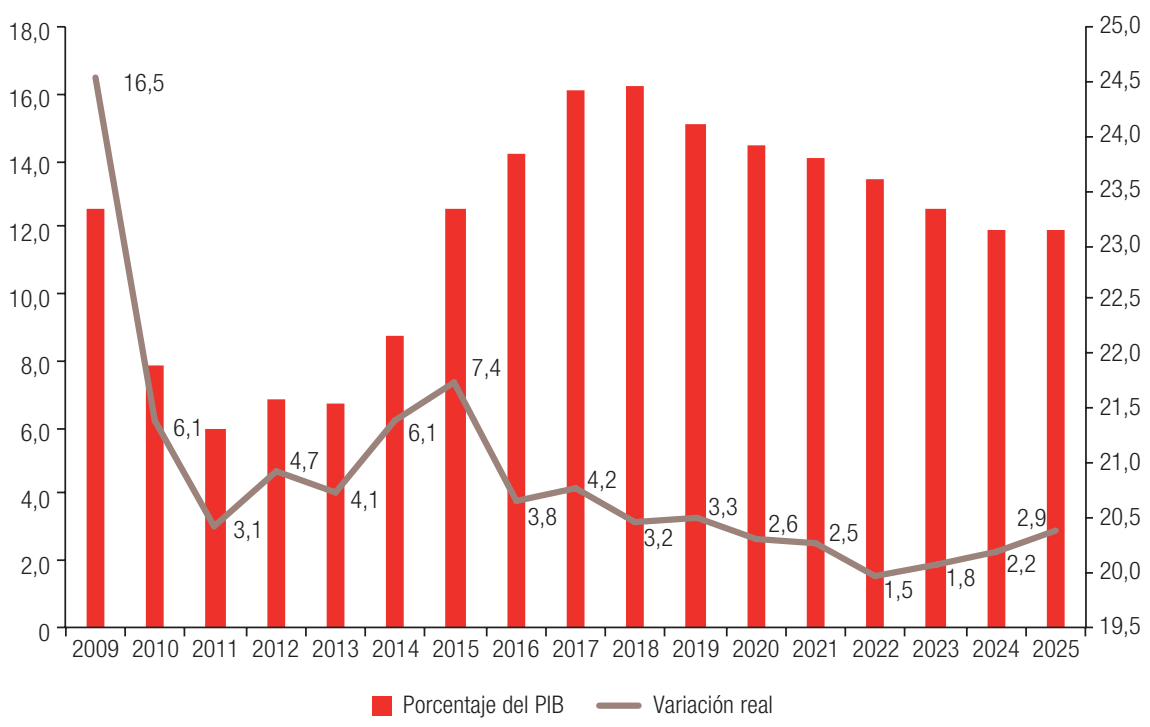

Fuente: Elaboración propia, sobre la base de información de la Dirección de Presupuestos (DIPRES) y el Ministerio de Hacienda.

\section{Cuadro 2}

Indicadores estáticos de vulnerabilidad y sostenibilidad fiscal, proyección 2018-2025ª escenario de tendencia

(En porcentajes)

\begin{tabular}{lcccc}
\hline Año & $\begin{array}{c}\text { (II) Deuda neta } \\
\text { (sin FRP) / PIB }\end{array}$ & $\begin{array}{c}\text { (II) Deuda neta } \\
\text { (con FRP) / PIB }\end{array}$ & $\begin{array}{c}\text { (VI) Balance primario } \\
\text { (metodología DIPRES) }\end{array}$ & $\begin{array}{c}\text { (VII) Balance primario } \\
\text { estructural }\end{array}$ \\
\hline 2018 & 8,9 & 5,0 & $-2,6$ & $-0,7$ \\
\hline 2019 & 10,1 & 6,2 & $-2,4$ & $-0,3$ \\
\hline 2020 & 11,0 & 6,9 & $-2,1$ & 0,0 \\
\hline 2021 & 11,4 & 7,2 & $-1,8$ & 0,2 \\
\hline 2022 & 11,3 & 7,1 & $-1,3$ & 0,5 \\
\hline 2023 & 11,0 & 6,7 & $-1,0$ & 0,7 \\
\hline 2025 & 10,5 & 6,2 & $-0,8$ & 0,8 \\
\hline
\end{tabular}

Fuente: Elaboración propia.

a Las cifras son consistentes con lo que se suponía al momento de la elaboración del Proyecto de Ley de Presupuestos de 2018.

La simulación muestra una deuda neta que tiende a equilibrarse en torno al $11 \%$ del PIB en caso de no considerarse el FRP y en torno al 7\% del PIB en caso de considerarse el FRP. En ambos casos se prevé una leve disminución de la deuda hacia el final del período. Por su parte, se proyectan repetidos déficits fiscales primarios efectivos, que al ser corregidos por el ciclo del producto mostrarían superávits estructurales primarios cercanos al 1\% del PIB hacia el final de la proyección.

En el gráfico 5 se exponen los resultados para el caso del escenario adverso ${ }^{24}$.

${ }^{24}$ Correspondiente a los resultados de suponer que se observe el escenario ii) del apartado b) de este documento. 


\section{Gráfico 5}

Escenario adverso

A. Deuda neta, sin incluir el Fondo de Reserva de Pensiones (FRP)

(en porcentajes y millones de pesos)

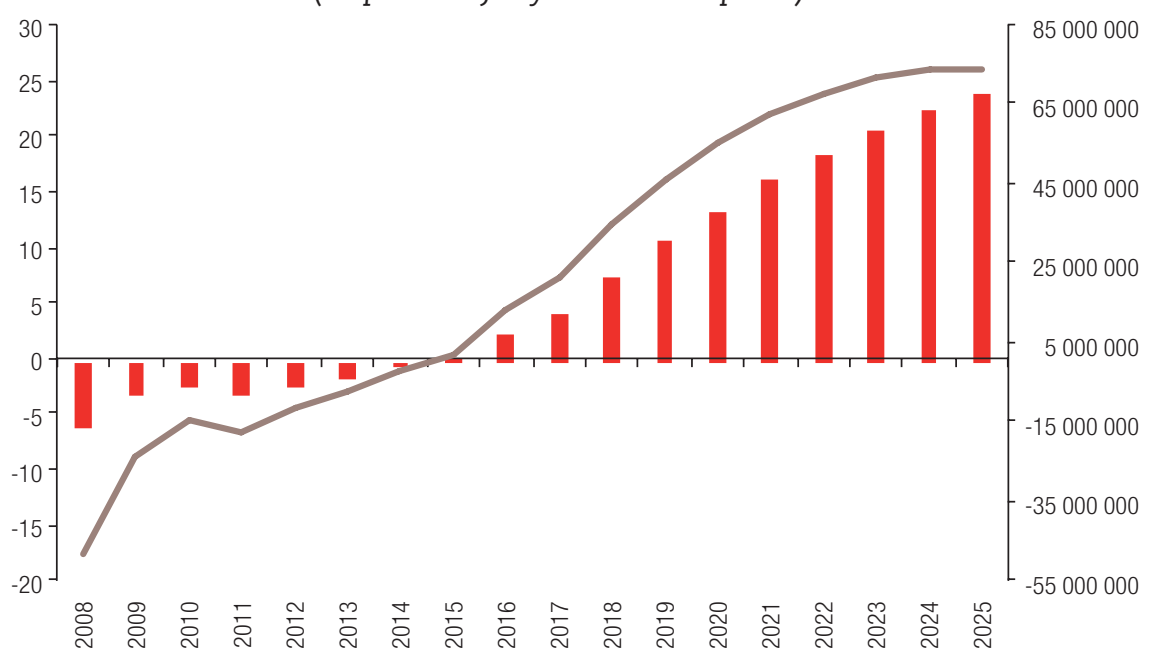

B. Deuda neta, incluido el Fondo de Reserva de Pensiones (FRP)

(en porcentajes y millones de pesos)

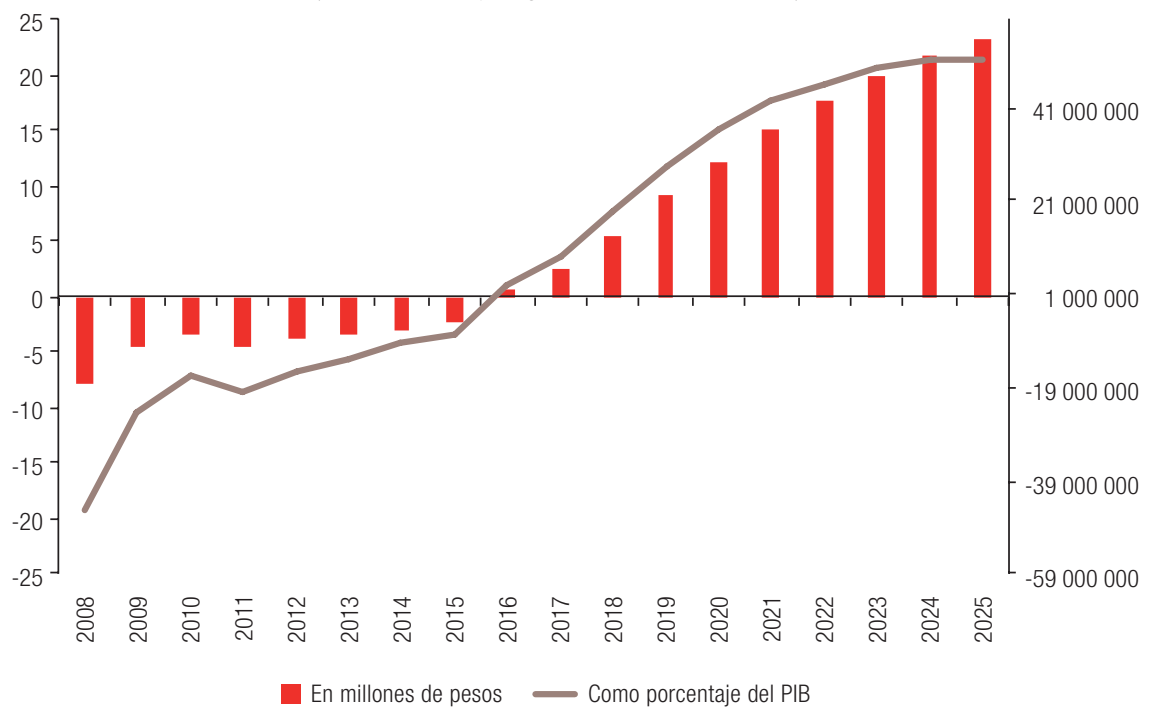

Fuente: Elaboración propia.

Como era de esperarse, en una situación adversa en términos de actividad, es necesario recurrir a un mayor endeudamiento (ya sea mediante más deuda bruta o por liquidación de activos). Se observa que un escenario de desaceleración importante de la actividad en 2018 podría llevar a la deuda neta a valores que superen el 20\% del PIB en 2021 y con una tendencia creciente hasta llegar al $26 \%$ del PIB hacia el final de la proyección. Esto obedece, principalmente, a que un escenario adverso como el descrito hace que el PIB efectivo se sitúe por debajo del PIB tendencial, lo que aumenta la brecha PIB. Así, el gobierno central se ve obligado a endeudarse o liquidar activos para poder cumplir con sus compromisos y, al mismo tiempo, alcanzar la meta del balance cíclicamente ajustado. 
Un supuesto importante es que la senda de metas del balance estructural es la misma que en el escenario de tendencia, lo que conlleva un menor espacio para el crecimiento del gasto. En el gráfico 6 se aprecia cuál sería la senda de crecimiento del gasto. Se observa una reducción paulatina del gasto como porcentaje del PIB a partir de 2019, lo que está de acuerdo con una meta de balance cíclicamente ajustado que tiende al equilibrio. En este escenario las variaciones reales del gasto se encontrarían en torno a un 1,2\% real anual.

\section{Gráfico 6}

Gasto total: crecimiento real y PIB en escenario adverso

(En porcentajes)

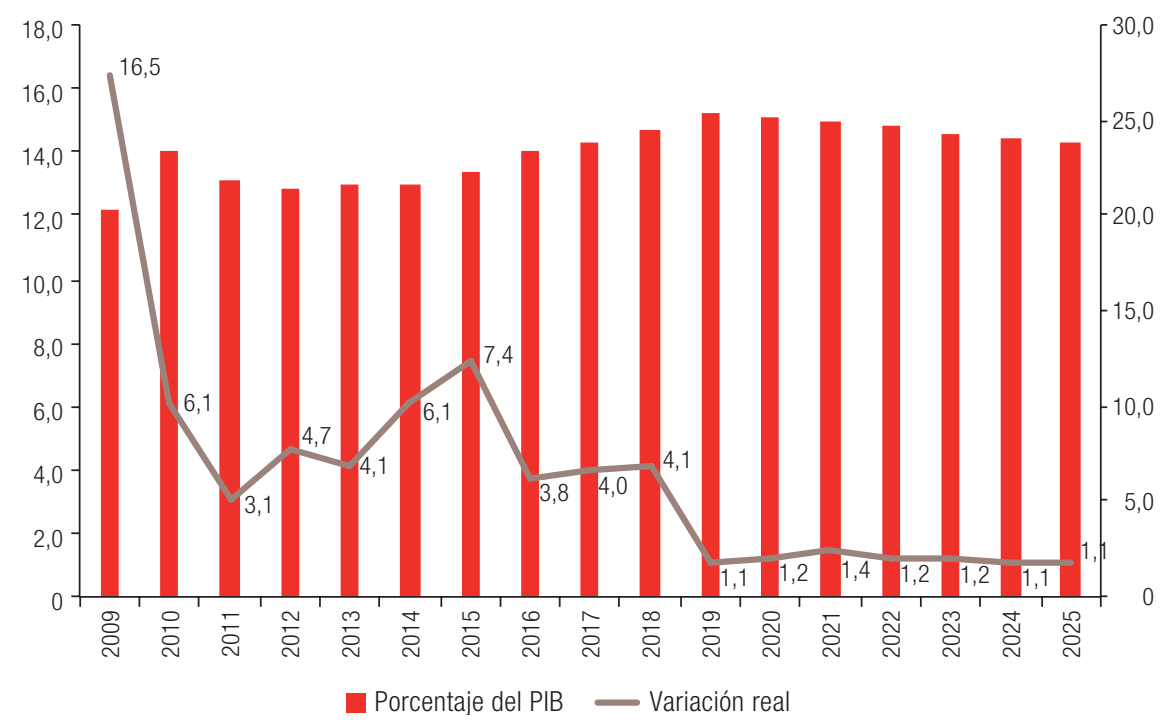

Fuente: Elaboración propia, sobre la base de información de la Dirección de Presupuestos (DIPRES) y el Ministerio de Hacienda.

Del mismo modo que lo realizado para el escenario de tendencia, en este caso se presenta la evolución de los indicadores simulados a 2025. De acuerdo con los indicadores evaluados (véase el cuadro 3), se observa un deterioro generalizado de la posición fiscal chilena. En todos los años de la proyección se registran déficits primarios efectivos. Por su parte, los déficits estructurales primarios son menores que los efectivos fruto del ajuste cíclico a los ingresos, que pasan a un superávit a partir de 2024.

\section{Cuadro 3}

Indicadores estáticos de vulnerabilidad y sostenibilidad fiscal, proyección 2018-2025a , escenario adverso

(En porcentajes)

\begin{tabular}{lcccc}
\hline Año & $\begin{array}{c}\text { (II) Deuda neta } \\
\text { (sin FRP) / PIB }\end{array}$ & $\begin{array}{c}\text { (II) Deuda neta } \\
\text { (con FRP) / PIB }\end{array}$ & $\begin{array}{c}\text { (VI) Balance primario } \\
\text { (metodología DIPRES) }\end{array}$ & $\begin{array}{c}\text { (VII) Balance primario } \\
\text { estructural }\end{array}$ \\
\hline 2018 & 12,0 & 7,5 & $-5,6$ & $-1,7$ \\
\hline 2019 & 16,0 & 11,7 & $-5,4$ & $-1,5$ \\
\hline 2020 & 19,4 & 15,0 & $-5,2$ & $-1,1$ \\
\hline 2021 & 22,0 & 17,5 & $-4,9$ & $-0,7$ \\
\hline 2022 & 23,8 & 19,3 & $-4,4$ & $-0,4$ \\
\hline 2023 & 25,1 & 20,5 & $-4,0$ & $-0,1$ \\
\hline 2024 & 25,8 & 21,2 & $-3,6$ & 0,2 \\
\hline
\end{tabular}

Fuente: Elaboración propia.

a Las cifras son consistentes con lo que se suponía al momento de la elaboración del Proyecto de Ley de Presupuestos de 2018. 


\section{Comentarios finales y resultados principales}

En diversos estudios, tanto a nivel nacional como internacional, se ha reconocido el comportamiento sistemáticamente ordenado que han exhibido las finanzas públicas de Chile.

Mediante la aplicación de indicadores de sostenibilidad fiscal se pudo inferir que la situación fiscal de Chile mejoró en forma sostenida hasta 2009. A partir de ese año, con la crisis financiera internacional, el terremoto y tsunami y las crecientes presiones por un mayor gasto fiscal se registró un deterioro de la posición fiscal chilena. De hecho, en 2016 el gobierno central pasa de ser un acreedor neto a deudor. Esta posición fiscal nos retrotrae a 2004, cuando la deuda neta representaba un 4\% del PIB, lo cual es consistente con el aumento que ha mostrado la deuda bruta, que en 2016 alcanzó un 21,3\% del PIB, su mayor nivel desde 1994. Este deterioro de las cuentas fiscales ha sido una de las razones por las que las clasificadoras de riesgo han decidido bajar la calificación del país de AA-, obtenida en 2012, a A+, en lo que constituye la primera rebaja en la nota del país en 25 años. No obstante, las cifras aún pueden ser consideradas saludables dentro del contexto internacional.

En la segunda parte de este documento se propone un modelo dinámico para evaluar la sostenibilidad fiscal del país en una perspectiva de largo plazo. En particular, este trabajo aporta a la literatura desarrollando un modelo ad hoc para la economía chilena, siendo el primero en incorporar la dinámica del FRP, cuyos intereses y reglas de acumulación y desacumulación son clave para proyectar los niveles de deuda del gobierno central. Además, se formaliza en el modelo empírico el cálculo del indicador de balance cíclicamente ajustado, aunque de manera simplificada, de acuerdo a los cambios más recientes realizados por la autoridad fiscal.

A fin de ejemplificar la operación del modelo dinámico se estimaron un escenario macroeconómico de tendencia y otro más adverso en términos de actividad. En el cuadro 4 figura un resumen de los resultados de la simulación.

Una conclusión de este ejercicio es que, considerando un escenario de tendencia basado en la Ley de Presupuestos de 2018, una política fiscal consistente con la regla de balance cíclicamente ajustado y siguiendo las metas de convergencia propuestas por la autoridad, Chile alcanzaría niveles de endeudamiento neto en torno al 10\% del PIB en 2025, para lo cual el gasto fiscal total debería ser en promedio un 2,5\% durante el período 2018-2025, todo esto considerando un crecimiento económico promedio del 3,2\% y un precio del cobre promedio de 2,9 dólares la libra en dicho período (véanse los supuestos macroeconómicos en el anexo A3). De acuerdo a nuestro análisis, en este caso Chile llegaría a un balance primario estructural en equilibrio en 2020, a un balance primario positivo en 2021 y a equilibrio en el balance estructural en 2024.

Por otro lado, considerando un escenario adverso, con un crecimiento promedio del 2,1\% y un precio del cobre promedio de 2,2 dólares la libra en el período analizado, el país llegaría a niveles de endeudamiento neto en torno al $26 \%$ del PIB en 2025. Para ello el gasto fiscal total debería ser en promedio un 1,6\% durante el período 2018-2025. Esto implicaría llegar a un balance primario estructural positivo de un $0,22 \%$ en 2024, a un balance primario de un $-3,14 \%$ en 2025 y a un balance estructural de un -1,2\% (en porcentajes del PIB) en 2025. Esta situación adversa dejaría al país con una situación fiscal muy parecida a la del comienzo de los años noventa, en condiciones de vulnerabilidad frente a nuevas crisis externas y con elevados gastos asociados a pagos del servicio de la deuda de gobierno, restando recursos importantes a la política social. Cabe recordar, por ejemplo, que entre 1990 y 1993 en Chile el pago de intereses era superior al gasto fiscal en educación y en salud (Arellano, 2017, pág. 13), lo cual es una realidad de muchos países de la región que no han logrado manejar adecuadamente su política fiscal. Es por esto que para Chile es clave respetar y profundizar su regla fiscal, instrumento de política económica muy exitosa que le ha permitido contar con una posición fiscal que es un ejemplo incluso para países de mayor nivel de desarrollo económico. 


\section{Cuadro 4}

Resultados sostenibilidad fiscal, proyección 2018-2025

(En porcentajes)

\begin{tabular}{lrrrrrrrr}
\hline Escenario de tendencia & 2018 & 2019 & 2020 & 2021 & 2022 & 2023 & 2024 & 2025 \\
\hline Deuda neta (sin FRP) (en porcentajes del PIB) & 8,94 & 10,14 & 10,96 & 11,35 & 11,27 & 10,97 & 10,54 & 10,14 \\
\hline Variación real del gasto total & 3,22 & 3,26 & 2,59 & 2,50 & 1,54 & 1,84 & 2,24 & 2,92 \\
\hline Balance primario (metodología DIPRES) & $-0,65$ & $-0,29$ & $-0,02$ & 0,23 & 0,47 & 0,70 & 0,81 & 0,77 \\
\hline Balance primario estructural & $-0,70$ & $-0,30$ & 0,00 & 0,20 & 0,50 & 0,70 & 0,80 & 0,80 \\
\hline Escenario adverso & 2018 & 2019 & 2020 & 2021 & 2022 & 2023 & 2024 & 2025 \\
\hline Deuda neta (sin FRP) (en porcentajes del PIB) & 11,98 & 16,03 & 19,40 & 21,97 & 23,77 & 25,05 & 25,80 & 26,06 \\
\hline Variación real del gasto total & 4,05 & 1,07 & 1,24 & 1,43 & 1,18 & 1,24 & 1,13 & 1,09 \\
\hline Balance primario (metodología DIPRES) & $-5,60$ & $-5,42$ & $-5,20$ & $-4,86$ & $-4,38$ & $-3,98$ & $-3,57$ & $-3,14$ \\
\hline Balance primario estructural & $-1,67$ & $-1,48$ & $-1,05$ & $-0,69$ & $-0,35$ & $-0,05$ & 0,22 & 0,47 \\
\hline
\end{tabular}

Fuente: Elaboración propia.

Por último, cabe destacar que el modelo desarrollado en este trabajo permite realizar múltiples simulaciones alternativas, cambiando los supuestos básicos y los escenarios a evaluar. Una limitación de este trabajo es que la metodología presentada no está incorporada a un modelo macro estándar intertemporal que permita analizar las virtudes de estabilización de este tipo de política fiscal en el tiempo y sus efectos en el crecimiento por separado, en comparación con políticas fiscales alternativas. Un buen punto de partida para realizar dicho estudio serían los trabajos de Kumhof y Laxton (2013) y Medina y Soto (2007), que en parte hacen dicho tipo de análisis pero no consideran las mejoras presentadas en este artículo, llámese incorporar la dinámica del FRP y realizar el cálculo del indicador de balance cíclicamente ajustado de acuerdo a los cambios más recientes introducidos por la autoridad fiscal chilena.

\section{Bibliografía}

Alberola, E. y J. Montero (2007), "Debt sustainability and procyclical fiscal policies in Latin America", Documentos de Trabajo, No 0611, Madrid, Banco de España.

Arellano, J. P. (2017), Panorama fiscal: propuestas frente a una situación amenazante, Santiago, Corporación de Estudios para Latinoamérica (CIEPLAN).

Arellano, S. y F. Larraín (1996), "Tipo de cambio real y gasto público: un modelo econométrico para Chile", Cuadernos de Economía, año 33, № 98, Santiago, Instituto de Economía, Pontificia Universidad Católica de Chile.

Arenas, A. y otros (2009), "Proyecciones fiscales del sistema de pensiones: bonos de reconocimiento y déficit operacional 2010-2050”, Estudios de Finanzas Públicas, № 14, Santiago, Dirección de Presupuestos (DIPRES).

Barnhill, T. y G. Kopits (2003), "Assessing fiscal sustainability under uncertainty", IMF Working Paper, № 03/79, Washington, D.C., Fondo Monetario Internacional (FMI).

BID (Banco Interamericano de Desarrollo) (2008), All That Glitters May Not Be Gold: Assessing Latin America's Recent Macroeconomic Performance, Washington, D.C., Departamento de Investigación [en línea] http:// idbdocs.iadb.org/wsdocs/getdocument.aspx?docnum=2246723.

Blanchard, O. (1990), "Suggestions for a new set of fiscal indicators", OECD Economics Department Working Paper, № 79, París, Organización de Cooperación y Desarrollo Económicos (OCDE).

Bohn, H. (2005), "The sustainability of fiscal policy in the United States", CESifo Working Paper, № 1446 [en línea] http://www.cesifo.de/DocDL/cesifo1_wp1446.pdf. (1998), "The behavior of U.S. public debt and deficits", The Quarterly Journal of Economics, vol. 113, № 3, Oxford University Press.

Borensztein, E. y otros (2010), "El manejo de los ingresos fiscales del cobre en Chile", Washington, D.C., Banco Interamericano de Desarrollo (BID). 
Bravo, J. y otros (2014), "Un modelo macroeconómico de proyección para Chile", Documento de Trabajo, $N^{\circ} 6$, Santiago, Ministerio de Hacienda.

Buiter, W. H. (1985), "A guide to public sector debt and deficits", Economic Policy, vol. 1, № 1.

Calvo, G. A., A. Izquierdo y E. Talvi (2003), "Sudden Stops, the Real Exchange Rate and Fiscal Sustainability: Argentina's Lessons", Banco Interamericano de Desarrollo (BID) [en línea] https://publications.iadb.org/ handle/11319/1909.

Caputo, R. y R. Valdés (2016), "A fiscal vaccine against the Dutch disease", Applied Economic Letters, vol. 23, No 1, Taylor \& Francis.

Chalk, N. y R. Hemming (2000), "Assessing fiscal sustainability in theory and practice", IMF Working Paper, $N^{\circ}$ 00/81, Washington, D.C., Fondo Monetario Internacional (FMI).

Chan-Lau, J. y otros (2010), "Chile: selected issues paper", IMF Country Report, № 10/299, Washington, D.C., Fondo Monetario Internacional (FMI).

Contreras, A. y otros (2008), "Activos financieros del tesoro público chileno: resultados y desafíos", Estudios de Finanzas Públicas, № 11, Santiago, Dirección de Presupuestos (DIPRES).

Corbo, V. y otros (2011), Propuestas para perfeccionar la regla fiscal [en línea] http://www. dipres.gob.cl/594/ articles-76544_doc_pdf.pdf.

Costa, R. y L. F. Lagos (2001), "Superávit estructural: ventajas y limitaciones", serie Informe Económico, $N^{\circ} 120$, Santiago, Libertad y Desarrollo.

Crispi, J. y A. Vega (2003), "Sostenibilidad y regla fiscal: análisis e indicadores para Chile", Estudios de Finanzas Públicas, № 3, Santiago, Dirección de Presupuestos (DIPRES).

Dabán, T. (2011), "Strengthening Chile's rule-based fiscal framework", IMF Working Paper, N WP/11/17, Washington, D.C., Fondo Monetario Internacional (FMI).

DIPRES (Dirección de Presupuestos) (2017a), Indicadores de balance cílcicamente ajustado. Metodología y resultados, 2016, Santiago.

- (2017b), Informe de finanzas públicas del proyecto de ley de presupuestos del sector público, 2018, Santiago.

(2017c), Evaluación de la gestión financiera del sector público en 2016 y actualización de proyecciones para 2017, Santiago.

_ (2017d), "Estadísticas de las finanzas públicas, estado de operaciones del gobierno central" [en línea] http://www.dipres.gob.cl/594/w3-propertyvalue-15407.html.

_(2013), "Evaluación de la gestión financiera del sector público en 2012 y actualización de proyecciones para 2013", Santiago [en línea] http://www.dipres.gob.cl/594/articles-104134_doc_pdf.pdf.

Ffrench-Davis, R. (2010), "Latin America: the structural fiscal balance in Chile, a move toward counter-cyclical macroeconomics", Journal of Globalization and Development, vol. 1, № 1, De Gruyter.

FMI (Fondo Monetario Internacional) (2010), "Staff guidance note on the application of the joint bank-fund debt sustainability framework for low-income countries", Policy Papers, Washington, D.C. (2008), "Sovereign wealth funds - A work program", Policy Papers, Washington, D.C.

(2003), "Sustainability Assessments - Review of Application and Methodological Refinements" [en línea] http://www.imf.org/external/np/pdr/sustain/2003/061003.pdf.

— (2002), "Assessing Sustainability" [en línea] http://www.imf.org/external/np/pdr/sus/2002/eng/052802.pdf. (2001), Manual de estadísticas de finanzas públicas, 2001 [en línea] https://www.imf.org/external/pubs/ $\mathrm{ft} / \mathrm{gfs} / \mathrm{manual} / \mathrm{esl} / \mathrm{pdf} / \mathrm{all}$.pdf.

Frankel, J. (2011), "A solution to fiscal procyclicality: the structural budget institutions pioneered by Chile", NBER Working Paper, № 16945, Cambridge, Massachusetts, Oficina Nacional de Investigaciones Económicas [en línea] www.nber.org/papers/w16945.pdf.

Gavin, M. y otros (1996), "Managing fiscal policy in Latin America and the Caribbean: volatility, procyclicality, and limited creditworthiness", Working Paper, № 326, Washington, D.C., Banco Interamericano de Desarrollo (BID).

Giorno, C. y otros (1995), "Potential output, output gaps and structural budget balances", OECD Economic Studies, № 24, París, Organización de Cooperación y Desarrollo Económicos (OCDE).

Hagemann, R. (1999), "The structural budget balance. The IMFs methodology", IMF Working Paper, N 99/95, Washington, D.C., Fondo Monetario Internacional (FMI).

Hamilton, J. D. y M. A. Flavin (1986), "On the limitations of government borrowing: a framework for empirical testing", American Economic Review, vol. 76, № 4, Nashville, Tennessee, American Economic Association. 
Ilzetzki, E. y C. A. Végh (2008), "Procyclical fiscal policy in developing countries: truth or fiction?", NBER Working Paper, № 14191, Cambridge, Massachusetts, Oficina Nacional de Investigaciones Económicas.

Kumhof, M. y D. Laxton (2013), "Simple fiscal policy rules for small open economies", Journal of International Economics, vol. 91, № 1, Amsterdam, Elsevier.

_(2009), "Chile’s structural fiscal surplus rule: a model-based evaluation", IMF Working Paper, № 09/88, Washington, D.C., Fondo Monetario Internacional (FMI).

Kunzel, P. y otros (2010), "Investment objectives of sovereign wealth funds: a shifting paradigm", Economics of Sovereign Wealth Funds: Issues for Policymakers, U. S. Das, A. Mazarei y H. van der Hoorn (eds.), Washington, D.C., Fondo Monetario Internacional (FMI).

Larch, M. y A. Turrini (2009), "The cyclically-adjusted budget balance in EU fiscal policy making: a love at first sight turned into a mature relationship", Economic Papers, № 374, Bruselas, Comisión Europea.

Larraín, F. y otros (2011), "Una política fiscal de balance estructural de segunda generación para Chile", Estudios de Finanzas Públicas, № 18, Santiago, Dirección de Presupuestos (DIPRES).

Larraín, F. y F. Parro (2006), "Chile menos volátil", Santiago, Instituto de Economía, Pontificia Universidad Católica de Chile [en línea] https://www.researchgate.net/publication/46547988_Chile_menos_volatil/fu Iltext/0e5f6fe8f0c4c08778fc5f89/46547988_Chile_menos_volatil.pdf.

Le Fort, G. (2006), "Política fiscal con meta estructural en la experiencia chilena", documento presentado en la Segunda Reunión Anual del Grupo Latinoamericano de Especialistas en Manejo de Deuda Pública (LAC Debt Group).

Ley, E. (2010), "Fiscal (and External) Sustainability", Banco Mundial [en línea] http://siteresources.worldbank. org/INTDEBTDEPT/Resources/468980-1207588563500/4864698-1207588597197/FSPrimer.pdf.

Marcel, M. (2010), "La regla de balance estructural en Chile: diez años, diez lecciones", Washington, D.C., Banco Interamericano del Desarrollo, inédito.

Medina, J. P. y C. Soto (2007), "Copper price, fiscal policy and business cycle in Chile", Documento de Trabajo, N ${ }^{\circ} 458$, Santiago, Banco Central de Chile.

Mendoza, E. G. y P. M. Oviedo (2009), "Public debt, fiscal solvency and macroeconomic uncertainty in Latin America. The cases of Brazil, Colombia, Costa Rica and Mexico", Economía Mexicana. Nueva Época, vol. 18, N2 2, Ciudad de México, Centro de Investigación y Docencia Económicas.

(2006), Fiscal Policy and Macroeconomic Uncertainty in Developing Countries: The Tale of the Tormented Insurer, Cambridge, Massachusetts, Oficina Nacional de Investigaciones Económicas.

OCDE (Organización de Cooperación y Desarrollo Económicos) (2010), Economic Survey of Chile 2010, París.

Restrepo, J. E. y C. Soto (2006), "Regularidades empíricas de la economía chilena: 1986-2005", Economía Chilena, vol. 9, № 2, Santiago, Banco Central de Chile.

Rodríguez, J., C. Tokman y A. Vega (2006), "Política de balance estructural: resultados y desafíos tras seis años de aplicación en Chile", Estudios de Finanzas Públicas, № 7, Santiago, Dirección de Presupuestos (DIPRES).

Schmidt-Hebbel, K. (2010), "Fiscal rules and institutions in the world and in Chile", Santiago, inédito.

Talvi, E. y C. A. Végh (2000), "La sostenibilidad de la política fiscal: un marco básico”, ¿Cómo armar el rompecabezas fiscal? Nuevos indicadores de sostenibilidad, E. Talvi y C. A. Végh (comps.), Washington, D.C., Banco Interamericano de Desarrollo (BID).

Tapia, H. (2015), "Fiscal rule in Chile: from 'automatic pilot' to 'navigation device'”, mayo, inédito.

Ter-Minassian, T. (2010), "Preconditions for a successful introduction of structural balance-based rules in Latin America and the Caribbean: a framework paper", Washington, D.C., Fondo Monetario Internacional (FMI), inédito.

Velasco, A. y otros (2010), "El enfoque de balance estructural en la política fiscal en Chile: resultados, metodología y aplicación al período 2006-2009", Estudios de Finanzas Públicas, № 15, Santiago, Dirección de Presupuestos (DIPRES).

Vergara, R. (2002), "Política y sostenibilidad fiscal en Chile", Estudios Públicos, № 88, Santiago, Centro de Estudios Públicos.

Walker, E. (2011), "Un asset allocation para el FEES”, Santiago, inédito [en línea] http://www.hacienda.cl/ english/sovereign-wealth-funds/relevant-studies/un-asset-allocation-para-el-fees.html. 


\section{Anexo A1}

\section{Indicadores de vulnerabilidad y sostenibilidad fiscal}

Deuda del gobierno (indicadores I y II)

$$
D_{t}=\frac{\text { Acervo de deuda }}{P I B_{t}}
$$

Indicador de servicio de la deuda (III y IV)

$$
\begin{aligned}
& S D_{t}=\frac{\text { Amortizaciones }_{t}+\text { Pago de intereses }_{t}}{\text { Ingreso corriente }_{t}} \\
& S D_{t}(\text { prueba ácida })=\frac{\text { Amortizaciones }_{t}+\text { Pago de intereses }_{t}}{\text { Ingreso corriente }_{t}-\text { Ingreso cobre }_{t}}
\end{aligned}
$$

Indicador del balance primario

a) Metodología tradicional FMI (indicador V)

$$
b p_{t}=\frac{\text { Ingresos totales }_{t}-\left(\text { Gastos totales }_{t}-\text { Pago de intereses }_{t}\right)}{\text { PIB }_{t}}
$$

b) Metodología DIPRES (indicadores VI y VII)

$$
b p_{t}=\frac{\left(\text { Ingresos totales }_{t}-\text { Ingresos de intereses }_{t}\right)-\left(\text { Gastos totales }_{t}-\text { Pago de intereses }_{t}\right)}{P I B_{t}}
$$




\section{Anexo A2}

\section{Detalle formal del modelo de sostenibilidad fiscal}

\section{Variación de la deuda neta como porcentaje del PIB}

Primero, la ecuación de Fisher se define de la siguiente manera:

$$
\left(1+r_{t}\right)=\left(1+i_{t}\right) /\left(1+\pi_{t}\right)
$$

Al dividir la deuda neta sobre el PIB nominal $\left(\mathrm{P}^{\star} \mathrm{Y}\right)$ del período $t$, se obtiene:

$$
\begin{gathered}
\frac{D_{t}}{P_{t} Y_{t}}=\frac{\left(1+i_{t}\right) \cdot D_{t-1}}{P_{t} Y_{t}}-\frac{B P_{t}}{P_{t} Y_{t}}-\frac{\Delta M_{t}}{P_{t} Y_{t}} \\
d_{t}=\frac{\left(1+i_{t}\right)}{\left(1+g_{t}\right)\left(1+\pi_{t}\right)} d_{t-1}-b p_{t}-\Delta m_{t} \\
\Rightarrow d_{t}=\frac{\left(1+r_{t}\right)}{\left(1+g_{t}\right)} d_{t-1}-b p_{t}-\Delta m_{t}
\end{gathered}
$$

Restando la deuda del período pasado como porcentaje del PIB en ambos lados de la ecuación, se tiene:

$$
\begin{gathered}
d_{t}-d_{t-1}=\Delta d_{t}=\frac{\left(1+r_{t}\right)}{\left(1+g_{t}\right)} d_{t-1}-d_{t-1}-b p_{t}-\Delta m_{t} \\
\Delta d_{t}=\frac{r_{t}-g_{t}}{1+g_{t}} d_{t-1}-b p_{t}-\Delta m_{t}
\end{gathered}
$$

\section{Descomposición: deuda interna y deuda externa, PIB transable y no transable}

La deuda del gobierno puede dividirse entre deuda interna (supra índice " $i$ ") y deuda externa (supra índice "e"). Cada tipo de deuda debe pagar intereses de acuerdo a la tasa de interés interna y externa, respectivamente. La ecuación (A9) incorpora a la ley de movimiento de la deuda esta descomposición.

$$
\begin{gathered}
D_{t}=D_{t}^{i}+e_{t} \cdot D_{t}^{e} \\
D_{t}=\left(1+i_{t}^{i}\right) D_{t-1}^{i}+e_{t}\left(1+i_{t}^{e}\right) D_{t-1}^{e}-B P_{t}-\Delta M_{t}
\end{gathered}
$$

Donde $e_{t}$ es el tipo de cambio (pesos por dólar) en el período $t$. Por otra parte, se definen las siguientes variables (Ley, 2010):

$$
\begin{gathered}
\alpha_{t}^{i}=\frac{D_{t}^{i}}{D_{t}} \\
\alpha_{t}^{e}=\frac{e_{t} \cdot D_{t}^{e}}{D_{t}} \\
\alpha_{t}^{i}+\alpha_{t}^{e}=1
\end{gathered}
$$


Así, es posible ordenar la ecuación (A9) para que quede expresada en los mismos términos que la ecuación (13) presentada en el cuerpo del documento.

$$
\begin{gathered}
D_{t}=\left(1+i_{t}^{i}\right) D_{t-1}^{i}+\left(1+i_{t}^{e}\right) e_{t} D_{t-1}^{e}-B P_{t}-\Delta M_{t} \\
D_{t}=\left[\left(1+i_{t}^{i}\right) \alpha_{t-1}^{i}+\left(1+i_{t}^{e}\right)\left(1+\varepsilon_{t}\right) \alpha_{t-1}^{e}\right] D_{t-1}-B P_{t}-\Delta M_{t} \\
D_{t}=\left[1+i_{t}^{i} \alpha_{t-1}^{i}+\alpha_{t-1}^{e}\left(i_{t}^{e}+\varepsilon_{t}+i_{t}^{e} \varepsilon_{t}\right)\right] D_{t-1}-B P_{t}-\Delta M_{t} \\
D_{t}=\left(1+i_{t}\right) D_{t-1}-B P_{t}-\Delta M_{t}
\end{gathered}
$$

Con $\varepsilon_{t}$ como la tasa de depreciación del tipo de cambio. Por su parte, la tasa de interés nominal en el período $t$ es igual a:

$$
i_{t}=i_{t}^{i} \alpha_{t-1}^{i}+\alpha_{t-1}^{e}\left(i_{t}^{e}+\varepsilon_{t}+i_{t}^{e} \varepsilon_{t}\right)
$$

Tal como es posible desagregar la deuda entre deuda interna y externa, el PIB se puede descomponer entre la producción transable (supra índice "i") y la producción no transable (supra índice " $e$ ") $)^{25}$.

$$
P_{t} Y_{t}=P_{t}^{i} Y_{t}^{i}+e_{t} P_{t}^{e} Y_{t}^{e}
$$

Definiendo las siguientes variables:

$$
\begin{gathered}
w_{t}^{i}=\frac{P_{t}^{i} Y_{t}^{i}}{P_{t} Y_{t}} \\
w_{t}^{e}=\frac{e_{t} P_{t}^{e} Y_{t}^{e}}{P_{t} Y_{t}} \\
g_{t}=\frac{\Delta Y_{t}}{Y_{t-1}}
\end{gathered}
$$

Y siguiendo un álgebra similar al caso de la deuda, es posible expresar el PIB nominal en el período $t$ en función del PIB nominal del período pasado:

$$
\begin{gathered}
P_{t} Y_{t}=\left(1+g_{t}\right)\left(1+w_{t-1}^{i} \pi_{t}^{i}+w_{t-1}^{e}\left(\pi_{t}^{e}+\varepsilon_{t}+\pi_{t}^{e} \varepsilon_{t}\right)\right) P_{t-1} Y_{t-1} \\
P_{t} Y_{t}=\left(1+g_{t}\right)\left(1+\pi_{t}\right) P_{t-1} Y_{t-1}
\end{gathered}
$$

Donde la inflación en $t$ se define como:

$$
\pi_{t}=w_{t-1}^{i} \pi_{t}^{i}+w_{t-1}^{e}\left(\pi_{t}^{e}+\varepsilon_{t}+\pi_{t}^{e} \varepsilon_{t}\right)
$$

${ }^{25}$ Véase la separación que proponen Restrepo y Soto (2006) para encontrar la proporción del PIB que es transable y la que es no transable. 


\section{Compilación del modelo de sostenibilidad para la economía chilena}

La variación de la deuda neta, separando al FRP de los activos de gobierno, queda descrita de la siguiente manera:

$$
\begin{gathered}
\Delta D_{t}=\Delta F R P_{t}-B E f_{t} \\
\Delta D_{t}=-B E f_{t}^{\prime} \\
\operatorname{con} B E f_{t}^{\prime}=B E f_{t}-\Delta F R P_{t}
\end{gathered}
$$

Se separa el FRP del resto de activos del gobierno por sus preestablecidas reglas de acumulación y desacumulación. Los aportes al FRP siguen la siguiente fórmula:

$$
A F R P_{t}=P_{t-1} Y_{t-1} * \min \left\{0,5 \% ; \max \left\{0,2 \% ; B E f_{t-1} / P_{t-1} Y_{t-1}\right\}\right\}
$$

Por lo que la variación del FRP después de 2016 queda definida de la forma:

$$
\begin{gathered}
F R P_{t}=F R P_{t-1}+A F R P_{t}-\frac{1}{3} *\left(\text { GPrev }_{t}-\text { GPrev }_{2008}\right) \\
F R P_{t}-F R P_{t-1}=A F R P_{t}-\frac{1}{3} *\left(\text { Grev }_{t}-\text { GPrev }_{2008}\right) \\
\Delta F R P_{t}=A F R P_{t}-\frac{1}{3} *\left(\text { GPrev }_{t}-\text { GPrev }_{2008}\right)
\end{gathered}
$$

Teniendo en cuenta lo anterior, la deuda como porcentaje del PIB se define:

$$
\begin{gathered}
\frac{D_{t}}{P_{t} Y_{t}}=\frac{\left(1+i_{t}\right) * D_{t-1}}{P_{t} Y_{t}}-\frac{B P_{t}^{\prime}}{P_{t} Y_{t}} \\
d_{t}=\frac{\left(1+i_{t}\right)}{\left(1+g_{t}\right)\left(1+\pi_{t}\right)} d_{t-1}-b p_{t}^{\prime} \\
d_{t}=\frac{\left(1+r_{t}\right)}{\left(1+g_{t}\right)} d_{t-1}-b p_{t}^{\prime}
\end{gathered}
$$

Y la variación de la deuda como porcentaje del PIB:

$$
\Delta d_{t}=\frac{r_{t} g_{t}}{1+g_{t}} d_{t-1}-b p_{t}^{\prime}
$$

Siguiendo la descomposición de Ley (2010), se definen las siguientes tasas de interés e inflación:

$$
\begin{gathered}
\hat{i}_{t}=\propto_{t-1}^{i} i_{t}^{i}+\propto_{t-1}^{e} i_{t}^{e} \\
\hat{\pi}_{t}=\omega_{t-1}^{i} \pi_{t}^{i}+\omega_{t-1}^{e} \pi_{t}^{e}
\end{gathered}
$$

Por lo que la tasa de interés nominal y la inflación quedan descritas de la forma:

$$
\begin{aligned}
i_{t} & =\hat{i}_{t}+\varepsilon_{t} \propto{ }_{t-1}^{e}\left(1+i_{t}^{e}\right) \\
\pi_{t} & =\hat{\pi}_{t}+\varepsilon_{t} w_{t-1}^{e}\left(1+\pi_{t}^{e}\right)
\end{aligned}
$$


Reemplazando en las ecuaciones (A10) y (A11) se obtiene:

$$
\begin{gathered}
d_{t}=\frac{\left(1+\hat{i}_{t}+\varepsilon_{t} \propto_{t-1}^{e}\left(1+i_{t}^{e}\right)\right)}{\left(1+g_{t}\right)\left(1+\hat{\pi}_{t}+\varepsilon_{t} w_{t-1}^{e}\left(1+\pi_{t}^{e}\right)\right)} d_{t-1}-b p_{t}^{\prime} \\
\Delta d_{t}=\left(\frac{\left(1+\hat{i}_{t}+\varepsilon_{t} \propto_{t-1}^{e}\left(1+i_{t}^{e}\right)\right)}{\left(1+g_{t}\right)\left(1+\hat{\pi}_{t}+\varepsilon_{t} w_{t-1}^{e}\left(1+\pi_{t}^{e}\right)\right)}-1\right) d_{t-1}-b p_{t}^{\prime} \\
\Delta d_{t}=\left(\begin{array}{c}
\left(1+r_{t}\right)=\frac{\left(1+\hat{i}_{t}+\varepsilon_{t} \propto_{t-1}^{e}\left(1+i_{t}^{e}\right)\right)}{\left(1+\hat{\pi}_{t}+\varepsilon_{t} w_{t-1}^{e}\left(1+\pi_{t}^{e}\right)\right)} \\
\frac{\left(\hat{i}_{t}-\hat{\pi}_{t}\right)+\varepsilon_{t}\left(\propto_{t-1}^{e}\left(1+i_{t}^{e}\right)-w_{t-1}^{e}\left(1+\pi_{t}^{e}\right)\right)}{\left(1+\hat{\pi}_{t}+\varepsilon_{t} w_{t-1}^{e}\left(1+\pi_{t}^{e}\right)\right)} \\
\left(1+\hat{\pi}_{t}+\varepsilon_{t} w_{t-1}^{e}\left(1+\pi_{t}^{e}\right)\right) \\
\left(1+g_{t}\right) \\
\Delta d_{t}=\frac{r_{t}-g_{t}}{1+g_{t}} d_{t-1}-b p_{t}^{\prime}
\end{array}\right) d_{t-1}-b p_{t}^{\prime}
\end{gathered}
$$

Definiendo entonces de esta manera la tasa de interés real. Por otra parte, si se quiere dejar la dinámica de la deuda en función del balance efectivo como porcentaje del PIB, se tiene:

$$
\begin{gathered}
\Delta d_{t}=\frac{r_{t}-g_{t}}{1+g_{t}} d_{t-1}-b p_{t}^{\prime} \\
\Delta d_{t}=\frac{i_{t}-\gamma_{t}}{1+\gamma_{t}} d_{t-1}-b p_{t}^{\prime} \\
\Delta d_{t}=\frac{i_{t}-\gamma_{t}}{1+\gamma_{t}} d_{t-1}-i_{t} d_{t-1}+i_{t} d_{t-1}-b p_{t}^{\prime} \\
\Delta d_{t}=\frac{i_{t}-\gamma_{t}}{1+\gamma_{t}} d_{t-1}-i_{t} d_{t-1}+i_{t} d_{t-1}+\frac{\Delta F R P_{t}}{P_{t} Y_{t}}-b p_{t} \\
\Delta d_{t}=\frac{1-i_{t}}{1+\gamma_{t}} \gamma_{t} d_{t-1}+\frac{\Delta F R P_{t}}{P_{t} Y_{t}}-b e f_{t}
\end{gathered}
$$

A su vez, el balance efectivo como porcentaje del PIB puede expresarse en función de la meta de balance cíclicamente ajustado:

$$
b e f_{t}=\frac{I T_{t}}{P_{t} Y_{t}}-\frac{G T_{t}}{P_{t} Y_{t}}
$$




$$
\begin{gathered}
\frac{G T_{t}}{P_{t} Y_{t}}=\frac{I T_{t}^{*}}{P_{t} Y_{t}}-\overline{b c a_{t}} \\
b e f_{t}=\frac{I T_{t}}{P_{t} Y_{t}}-\left(\frac{I T_{t}^{*}}{P_{t} Y_{t}}-\overline{b c a_{t}}\right) \\
\Rightarrow b e f_{t}=\overline{b c a_{t}}-a c_{t}
\end{gathered}
$$

Así, reemplazando el término de balance efectivo, es posible dejar la ecuación de dinámica de la deuda en función de la meta de balance estructural:

$$
\Delta d_{t}=-\frac{1+i_{t}}{1+\gamma_{t}} \gamma_{t} d_{t-1}+\frac{\Delta F R P_{t}}{P_{t} Y_{t}}-\overline{b c a_{t}}-a c_{t}
$$

Donde $a c_{t}$, el ajuste cíclico como porcentaje del PIB, se calcula de la siguiente manera:

$$
\begin{gathered}
a c_{t}=\frac{I T N M_{t}}{P_{t} Y_{t}} \cdot\left(1-\left(\frac{Y_{t}^{*}}{Y_{t}}\right)^{n}\right) \\
+\frac{\left(V C_{t} \cdot\left(P_{t}^{C}-P_{t}^{*}\right)+V M_{t} \cdot\left(P M_{t}-P M_{t}^{*}\right)\right) \cdot e_{t} \cdot 2.204,62}{P_{t} Y_{t}} \\
+\frac{\left(P_{t}^{B M L}-P_{t}^{*}\right) \cdot \tilde{\tau}_{t} \cdot Q_{t} \cdot e_{t} \cdot 2.204,62}{P_{t} Y_{t}}
\end{gathered}
$$




\section{Anexo A3}

\section{Escenarios macroeconómicos simulados}

Ambos escenarios toman como base lo publicado en el Informe de Finanzas Públicas de octubre de 2018. Por lo tanto, aunque en ese informe había proyecciones de cifras cuyo valor efectivo a la fecha ya es conocido, al ser información de dominio público permite una mejor replicabilidad del ejercicio.

Cuadro A3.1

Escenario de tendencia

(En porcentajes)

\begin{tabular}{|c|c|c|c|c|c|c|c|c|}
\hline & 2018 & 2019 & 2020 & 2021 & 2022 & 2023 & 2024 & 2025 \\
\hline \multicolumn{9}{|l|}{$\mathrm{PIB}$} \\
\hline Tasa de variación real (PIB efectivo) & 3,0 & 3,3 & 3,5 & 3,5 & 3,4 & 3,0 & 3,0 & 3,0 \\
\hline Tasa de variación real (PIB tendencia) & 2,6 & 2,7 & 2,8 & 2,9 & 3,0 & 3,0 & 3,0 & 3,0 \\
\hline Brecha PIB & 2,2 & 1,7 & 1,0 & 0,4 & 0,0 & 0,0 & 0,0 & 0,0 \\
\hline \multicolumn{9}{|l|}{ IPC } \\
\hline Variación (prom/prom) & 2,6 & 3,0 & 3,0 & 3,0 & 3,0 & 3,0 & 3,0 & 3,0 \\
\hline \multicolumn{9}{|l|}{ Tipo de cambio peso/dólar } \\
\hline Valor nominal & 650 & 650 & 650 & 650 & 650 & 650 & 650 & 650 \\
\hline \multicolumn{9}{|l|}{ Precio del cobre en dólares la libra } \\
\hline Valor nominal Bolsa de Metales de Londres & 2,9 & 2,8 & 2,8 & 2,8 & 2,9 & 2,9 & 2,9 & 2,9 \\
\hline Precio de referencia & 2,8 & 2,8 & 2,8 & 2,8 & 2,8 & 2,8 & 2,8 & 2,8 \\
\hline \multicolumn{9}{|l|}{ Variables fiscales } \\
\hline Meta de balance estructural (en porcentajes del PIB) & $-1,4$ & $-1,2$ & $-0,9$ & $-0,7$ & $-0,4$ & $-0,2$ & 0,0 & 0,0 \\
\hline
\end{tabular}

Cuadro A3.2

Escenario adverso

(En porcentajes)

\begin{tabular}{|c|c|c|c|c|c|c|c|c|}
\hline & 2018 & 2019 & 2020 & 2021 & 2022 & 2023 & 2024 & 2025 \\
\hline \multicolumn{9}{|l|}{ PIB } \\
\hline Tasa de variación real (PIB efectivo) & 0,5 & 1,5 & 2,0 & 2,5 & 2,8 & 2,5 & 2,5 & 2,5 \\
\hline Tasa de variación real (PIB tendencia) & 2,0 & 2,0 & 2,0 & 2,0 & 2,0 & 2,0 & 2,0 & 2,0 \\
\hline Brecha PIB & 4,0 & 4,6 & 4,6 & 4,1 & 3,3 & 2,8 & 2,3 & 1,8 \\
\hline \multicolumn{9}{|l|}{ IPC } \\
\hline Variación (prom/prom) & 0,04 & 0,02 & 0,025 & 0,03 & 0,03 & 0,03 & 0,03 & 0,03 \\
\hline \multicolumn{9}{|l|}{ Tipo de cambio peso/dólar } \\
\hline Valor nominal & 750 & 700 & 695 & 690 & 685 & 680 & 675 & 670 \\
\hline \multicolumn{9}{|l|}{ Precio del cobre en dólares la libra } \\
\hline Valor nominal Bolsa de Metales de Londres & 1,5 & 2,0 & 2,1 & 2,2 & 2,3 & 2,4 & 2,5 & 2,6 \\
\hline Precio de referencia & 2,3 & 2,3 & 2,3 & 2,3 & 2,3 & 2,3 & 2,3 & 2,3 \\
\hline \multicolumn{9}{|l|}{ Variables fiscales } \\
\hline Meta de balance estructural (en porcentajes del PIB) & $-2,5$ & $-2,7$ & $-2,5$ & $-2,2$ & $-2,0$ & $-1,7$ & $-1,5$ & $-1,2$ \\
\hline
\end{tabular}

Fuente: Elaboración propia, sobre la base de Dirección de Presupuestos (DIPRES), Informe de finanzas públicas del proyecto de ley de presupuestos del sector público, 2018, Santiago, 2017. 